\title{
Intramolecular Charge Transfer in the Azathioprine Prodrug Quenches Intersystem Crossing to the Reactive Triplet State in 6-Mercaptopurine
}

Luis A. Ortiz-Rodríguez, ${ }^{1}$ Glesmarie Ortiz-Zayas, ${ }^{1}$ Marvin Pollum, ${ }^{1}$ Sean J. Hoehn, ${ }^{1}$ Steffen Jockusch, ${ }^{2}$ Carlos E. Crespo-Hernández ${ }^{* 1}$

${ }^{1}$ Department of Chemistry, Case Western Reserve University, Cleveland, OH 44106

${ }^{2}$ Department of Chemistry, Columbia University, New York, NY 10027

* Corresponding author; E-mail: carlos.crespo@ case.edu; ORCID: 0000-0002-3594-0890

Submitted for consideration for the Special Issue Honoring Jean Cadet

TOC
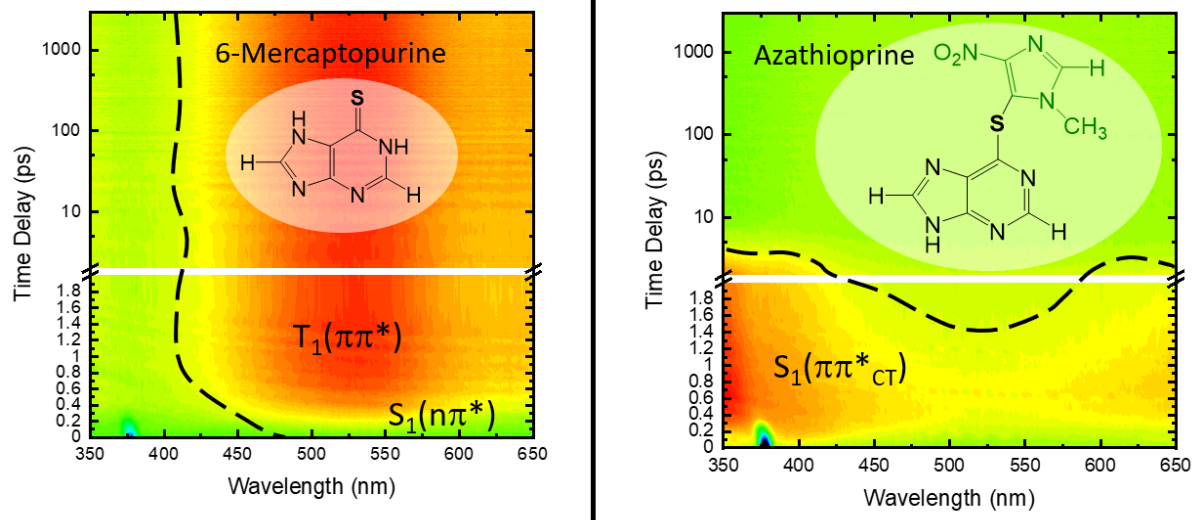


\begin{abstract}
The thiopurine prodrugs 6-mercaptopurine and azathioprine are among the world's essential medications for acute lymphoblastic leukemia, immunosuppression, and several autoimmune conditions. Thiopurine prodrugs are efficient UVA absorbers and singlet oxygen generators and the long-term treatment with these prodrugs correlates with a high incidence of sunlight-induced skin cancer in patients. In this contribution, we show that the electronic relaxation mechanisms and photochemical properties of azathioprine are remarkably different from those of 6mercaptopurine upon absorption of UVA radiation. UVA excitation of 6-mercaptopurine results in nearly $100 \%$ triplet yield and up to $30 \%$ singlet oxygen generation, whereas excitation of azathioprine with UVA leads to triplet yields of 15 to $3 \%$ depending on $\mathrm{pH}$ of the aqueous solution and less than $1 \%$ singlet oxygen generation. While photoexcitation of 6-mercaptopurine and other thiopurine prodrugs can facilitate oxidatively generated cell damage, azathioprine's poor photosensitization ability reveals the use of interchromophoric charge transfer interactions for the molecular design of photostable prodrugs exhibiting a remarkable reduction in photocytotoxic side effects before drug metabolization.
\end{abstract}




\section{INTRODUCTION}

Thiopurine prodrugs azathioprine (Aza) and 6-mercaptopurine (6MP) figure in the World Health Organization top one hundred list of essential medicines and are prescribed for the treatment of leukemia, autoimmune conditions, and as immunosuppressants. ${ }^{1}$ Although they are highly effective medications, long-term treatment of patients with thiopurine prodrugs is associated with a 10-fold increase in basal cell carcinomas and a 65 - to 250-fold increase in squamous cell carcinomas. ${ }^{2}$ These side effects are induced by exposure to sunlight, ${ }^{3-5}$ which is thought to originate from the ability of thiopurine derivatives to absorb UVA radiation (320-400 $\mathrm{nm}) .{ }^{6}$ Indeed, UVA irradiation of cells treated with Aza and 6MP causes photochemical damage, increasing oxidatively generated damage of both the genome and the proteome. ${ }^{7,} 8$ The photochemical properties of 6-thioguanosine (6tGuo), a clinically-active metabolite of these prodrugs, ${ }^{3,9,10}$ have received widespread attention ${ }^{6,11-18}$ because is thought to be the root cause of the mutagenic damage.

Recent investigations have shown that approximately half of the observed oxidatively generated DNA damage in cells treated with Aza or 6-thioguanine (6tGua) originates from the direct photoactivation of the metabolized 6 tGuo nucleotide in the intracellular pool. ${ }^{9}$ This raises an important question as to whether Aza and 6MP themselves can induce oxidatively generated damage to cellular DNA in the intracellular pool and to the proteome. To narrow this knowledge gap, a thorough investigation of the photochemical properties of both drugs in aqueous solutions under physiological-relevant conditions must be performed. Such a study can reveal the phototoxic likelihood of these drugs to cells and if it is necessary to investigate their direct participation in UVA-induced cellular damage. Indeed, UVA-excited 6tGua has been shown to induce oxidatively generated damage to nucleotides in the intracellular pool, ${ }^{9}$ forming the 2 '- 
deoxy-8-oxoguanosine nucleotide, which is then incorporated into cellular DNA, ${ }^{19}$ leading to indirect DNA damage.

In this contribution, we show that 6MP and Aza prodrugs exhibit remarkably different photochemical properties and electronic relaxation mechanisms. As observed for all other thiobases investigated to date, ${ }^{18,20-22}$ UVA excitation of 6MP leads to near-unity triplet yield and relatively-high singlet oxygen $\left({ }^{1} \mathrm{O}_{2}\right)$ generation. Conversely, excitation of Aza leads to triplet yields in the range of 15 to $3 \%$ depending on the protonation status of Aza and less than $1 \%{ }^{1} \mathrm{O}_{2}$ generation, which should lead to a significant reduction in photocytotoxic side effects before drug metabolization.

\section{EXPERIMENTAL AND THEORETICAL METHODS}

Chemicals. Azathioprine, and 6-mercaptopurine monohydrate were obtained from SigmaAldrich and used as received. 6-Thioguanosine, 2-amino-6-mercapto-9-( $\beta$-Dribofuranosyl)purine, was obtained from Carbosynth and used as received. Phosphate buffer saline (PBS) solutions were prepared freshly by mixing $0.2213 \mathrm{~g}$ disodium hydrogen phosphate (Sigma-Aldrich) and $0.3000 \mathrm{~g}$ sodium dihydrogen (Sigma-Aldrich) in $250 \mathrm{~mL}$ ultrapure water. The borate buffer at $\mathrm{pH} 9.8$ was prepared by dissolving $3.0661 \mathrm{~g}$ of disodium tetra-borate decahydrate (Sigma-Aldrich) in $500 \mathrm{~mL}$ of ultrapure water. The $\mathrm{pH}$ was adjusted using a $2 \mathrm{M}$ sodium hydroxide solution. HPLC grade acetonitrile (Acetonitrile) was obtained from Fisher Scientific and used as received.

Steady-State Measurements. Steady-state absorption and emission were measured at room temperature using a Carry Bio 300 spectrophotometer (Varian, Inc.) and a Cary Eclipse fluorescence spectrophotometer (Varian, Inc.), respectively. The molar extinction coefficients 
were determined from the slope of an absorbance versus concentration plot using a series of solutions of known concentration. Luminescence data for air- and $\mathrm{N}_{2}$-saturated aqueous solutions was collected every $0.5 \mathrm{~nm}$ with a $2 \mathrm{~s}$ averaging time, excitation and emission slit widths set to 5 $\mathrm{nm}$, and the PMT voltage set to $800 \mathrm{~V}$.

Electronic-Structure Calculations and Determination of Thermodynamic Values. Groundand excited state optimizations and vertical excitation energies were performed using Gaussian 16 suite of programs. Spin-orbit coupling constants (SOCs) and excited state absorption spectra were calculated with ORCA 4.2.1. Implicit solvation effects were modeled in Gaussian 16 or in ORCA 4.2.1 using the integral equation formalism of the polarizable continuum model $(\text { IEFPCM })^{23,}{ }^{24}$ or the conductor-like polarizable continuum model (CPCM), ${ }^{25}$ respectively. Solvation effects were also modeled explicitly in the ground state optimizations, vertical excitation energies, and spin-orbit coupling calculations by adding water molecules that form hydrogen bonds with the nitrogen atoms of the purine chromophore.

The ground state geometries of 6MP and Aza were optimized in water (IEFPCM + water molecules) by implementation of the density functional theory (DFT) using the B3LYP functional $^{26,27}$ and the $6-311+\mathrm{G}(\mathrm{d}, \mathrm{p})$ standard basis set using Gaussian 16 suite of programs. Single-point calculations were also performed in the DFT-optimized ground state structure at the MP2 level of theory to calculate the relative energies among possible tautomers. The optimized ground state geometries were then used to calculate the vertical excitation energies (VEEs) and SOCs in water by implementation of the time-dependent DFT (TD-DFT) using the CAM$\mathrm{B} 3 \mathrm{LYP},{ }^{28} \omega \mathrm{B} 97 \mathrm{X}^{29}$ and $\mathrm{PBE}^{23,}{ }^{24}$ functionals and the $6-311+\mathrm{G}(\mathrm{d}, \mathrm{p})$ basis set at TD-DFT level of theory. The character of each vertical transition and optimized structures was assigned based 
the magnitudes of the oscillator strengths and by visual inspection of the Kohn-Sham orbitals. The excited states of singlet and triplet state were optimized in water using the TDPBE0/IEFPCM/6-311+G(d,p) level of theory. The lowest-energy triplet state of 6mercaptopurine was also optimized using the same functional and basis set used in the TD-DFT optimizations but using unrestricted DFT and charge and multiplicity of 0 and 3 , respectively. All geometries were optimized without constraints.

The energy difference between the triplet and the ground state optimized geometries is reported as the adiabatic energy of the lowest-energy triplet state of 6MP. The oxidation potential $\left(\mathrm{E}_{\mathrm{ox}}{ }^{\circ}\right)$ of $6 \mathrm{MP}$ was estimated using equation (1) and the vertical ionization energy (VIE) of 6MP using the methodology described by Crespo-Hernández and co-workers. ${ }^{30}$ Initially, the ground state geometries for the neutral and deprotonated forms of 6MP were optimized at the B3LYP/6-31++G(d,p) level of theory. The optimized ground state geometries were then used to obtain the VIEs at the UB3LYP/6-31++G(d,p) level of theory. ${ }^{30}$ This simple method has been previously used to determine the redox potentials of the DNA canonical bases, ${ }^{30}$ 6thioguanosine, ${ }^{16}$ and other nucleobase derivatives. ${ }^{31,32}$

$$
E_{o x}^{\circ}=(-2.59 \pm 0.26)+(0.56 \pm 0.03) \times V I E
$$

The Rehm-Weller equation $(2)^{33}$ was then used to estimate the Gibbs free energy for electron transfer $\left(\Delta \mathrm{G}_{\mathrm{ET}}\right)$ between the lowest-energy triplet state of $6 \mathrm{MP}$ and $\mathrm{O}_{2}$ :

$$
\Delta G_{E T}=E_{o x}^{6 M P}-E_{\text {red }}^{O_{2}}-E_{T}^{6 M P}-C
$$

where $E_{o x}^{6 M P}$ is the oxidation potential of $6 \mathrm{MP}$ determined using equation (1), $E_{\text {red }}^{O_{2}}$ is the reduction potential of ground state $\mathrm{O}_{2}, E_{T}^{6 M P}$ is the adiabatic energy of the lowest-energy triplet state of $6 \mathrm{MP}$, and $\mathrm{C}$ is the Coulombic interaction term experienced by the ion pair following the electron transfer process. ${ }^{34}$ The latter term represents a small contribution to the overall energy 
change for neutral species in polar solvents ${ }^{35}$ and has approximated to be $0.06 \mathrm{eV}$ in acetonitrile. ${ }^{34}$ The reduction potential of ground state $\mathrm{O}_{2}$ has been reported to be $-51.7 \mathrm{~kJ} / \mathrm{mol} .{ }^{36}$

Femtosecond Broadband Transient Absorption Spectroscopy. Transient absorption spectroscopy with femtosecond time resolution was used to study the ultrafast excited-state dynamics of Aza and 6MP. The procedure used is detailed elsewhere. ${ }^{37-39}$ Briefly, a Ti-Sapphire amplifier laser system (Libra-He, Coherent, Inc.) generates $4.0 \mathrm{~W}, 100 \mathrm{fs}$ pulses at $800 \mathrm{~nm}$ and a repetition rate of $1 \mathrm{kHz}$. The fundamental laser beam was split into two distinct beams using a beam splitter. One of the beams was used to pump an optical parametric amplifier (OPA, TOPAS, Quantronix/Light Conversion), of which the output was tuned to the excitation wavelengths of $350 \mathrm{~nm}, 334 \mathrm{~nm}$, or $316 \mathrm{~nm}$ and attenuated to $1 \mu \mathrm{J}$ at the sample using a neutral density optical filter. The other fraction of the fundamental beam from the Libra-He was used to pump a constantly translating $\mathrm{CaF}_{2}$ crystal of $2 \mathrm{~mm}$ thickness to generate the white light continuum (WLC) to be used as the probe beam $(320-710 \mathrm{~nm})$. A Helios spectrometer (Ultrafast Systems, LLC.) was used, with a mechanical delay stage of a maximum of 3 ns for the probe pulse. This probe pulse was split into two fractions before reaching the sample. One fraction goes through the sample ( $2 \mathrm{~mm}$ path length) while the other fraction is used as reference, to account for changes in the WLC throughout the course of the experiment. The reference signal is generated via a chopper wheel, which blocks every other pump pulse. This provides an alternating sequence of spectra with and without sample excitation, thus allowing for the collection of $\Delta \mathrm{A}$ transient absorption data. Detection is achieved by placing two complementary metal-oxide-semiconductor (CMOS) detectors, one for the probe fraction that goes through the sample beam and one for the probe reference beam. 
All solutions were freshly prepared and were continuously stirred through the entire length of the experiment in a $2 \mathrm{~mm}$ optical path length quartz cell (Starna Cells, Inc.) using a Teflon-coated magnetic stirring bar. The sample was replaced with a fresh solution to maintain less than five percent decrease in absorbance through the entire length of the experiment, as monitored from the maxima of the lowest-energy band of the absorption spectra.

The transient absorption data was collected using a homemade software (LabView, National Instruments, Inc.). Data was corrected prior to analysis for group velocity dispersion (GVD) of the WLC using the two-photon absorption signal of neat methanol. Global and target analyses of the femtosecond transient absorption data was done using the Glotaran software were a two-component sequential model was used. Time zero was determined from the Raman stimulated emission from the solvent centered around $395 \mathrm{~nm}$ (when using PBS and $390 \mathrm{~nm}$ when using acetonitrile, and exciting at $350 \mathrm{~nm} ; \quad 355 \mathrm{~nm}$ using PBS and $~ 350 \mathrm{~nm}$ using acetonitrile, and exciting at $316 \mathrm{~nm} ; \quad 375 \mathrm{~nm}$ using PBS and exciting at $334 \mathrm{~nm}$ ). This band at its maximum amplitude was used to set time zero because it represents the maximum overlap of the pump and probe pulses. The uncertainties in the lifetimes are reported as twice the standard deviation $(2 \sigma)$ obtained from the global fit analysis using the Glotaran software. The percentage of the relative triplet yield for Aza were estimated by using equation (3):

$$
\Phi_{T}=\frac{\left|A_{\tau_{2}}\right|}{\left|A_{\tau_{1}}\right|+\left|A_{\tau_{2}}\right|} \times 100
$$

where $A_{\tau_{1}}$ and $A_{\tau_{2}}$ are the integrated area under the evolution-associated difference spectra obtained for $\tau_{1}$ and $\tau_{2}$, respectively, from the global analysis.

Nanosecond Broadband Transient Absorption Spectroscopy. Nanosecond broadband transient experiments were conducted to observe the dynamics of 6MP after the 3 ns limit of the 
mechanical delay stage of the femtosecond Helios spectrometer (Ultrafast Systems, LLC). Its methodology and experimental setup have been previously detailed. ${ }^{37}$ In brief, the same Ti:Sapphire amplifier system (Libra-HE, Coherent, Inc) was used to pump the OPA (TOPAS, Quantronix/Light Conversion) and generate the excitation wavelength of $334 \mathrm{~nm}$ used for the experiments. An electronically triggered broadband white light source (Eos, Ultrafast Systems, LLC) was used to generate a probe pulse with a spectral window from 375 to $800 \mathrm{~nm}$, a temporal window of up to $90 \mu \mathrm{s}$ and a time resolution of $400 \mathrm{ps}$. This light source was coupled to the Helios spectrometer and the data was collected using a homemade LabView (National Instruments, Inc.) software.

$\mathrm{N}_{2}$-saturated solutions of 6MP were prepared in PBS $\mathrm{pH} 7.4$ and 5.5 with phosphate concentration of $16 \mathrm{mM}$ and transient absorption data were collected in $2 \mathrm{~mm}$ optical quartz cells. Solutions were continuously stirred with a Teflon-coated magnetic stir bar. The sample was replaced with a fresh solution to maintain less than five percent decrease in absorbance through the entire length of the experiment, as monitored from the steady-state absorption spectra. Forty kinetic traces were extracted from each data set for target analysis using the Igor Pro 6.3.7.2 software (Wavemetrics, Inc.). The pH 5.5 sample was fitted to a single-exponential function, while $\mathrm{pH} 7.4$ sample was fitted to a two-exponential function. Both fit functions were convoluted with an instrument response function of $400 \mathrm{ps}$. The uncertainties in the lifetimes are reported as twice the standard deviation $(2 \sigma)$ obtained from the target analysis using the Igor Pro software.

Triplet-triplet Extinction Coefficient and Triplet Yield. The extinction coefficient for 6MP's triplet-triplet absorption band was calculated using the singlet depletion method. ${ }^{40}$ This method, 
detailed in reference, ${ }^{30}$ is convenient for thiobases and has been previously used for 4thiothymidine, ${ }^{41}$ 4-thiouracil, ${ }^{42}$ and 6-thioguanosine. ${ }^{13}$ Briefly, the excited triplet state concentration of $6 \mathrm{MP},\left[{ }^{3} \mathrm{M}^{*}\right]$, can be obtained for any given time delay using equation (4):

$$
\Delta A_{G S}\left(\lambda_{1}\right)=-\varepsilon_{G S}\left(\lambda_{1}\right)\left[{ }^{3} M^{*}\right] l
$$

where $\Delta A_{G S}\left(\lambda_{1}\right)$ is the intensity of the ground state bleaching signal of $6 \mathrm{MP}$ at $\lambda_{1}, \varepsilon_{G S}\left(\lambda_{1}\right)$ is the molar extinction coefficient of the ground state of 6MP at $\lambda_{1}$, and $l$ is the path length of the cuvette $(2 \mathrm{~mm})$. The triplet-triplet extinction coefficient of $6 \mathrm{MP}$ at $\lambda_{2}, \varepsilon_{T-T}{ }^{*}\left(\lambda_{2}\right)$, can be determined using equation (5):

$$
\Delta A_{T}\left(\lambda_{2}\right)=\varepsilon_{T-T}^{*}\left(\lambda_{2}\right)\left[{ }^{3} M^{*}\right] l
$$

where $\Delta A_{T}\left(\lambda_{2}\right)$ is the absorption intensity of the triplet band of $6 \mathrm{MP}$ at $\lambda_{2}$. The wavelength of the triplet-triplet absorption band $\left(\lambda_{2}\right)$ must be well-separated from the ground state bleaching $\operatorname{signal}\left(\lambda_{1}\right)$.

Having determined $\varepsilon_{T-T}{ }^{*}\left(\lambda_{2}\right)$ for $6 \mathrm{MP}$, the relative actinometry method ${ }^{40,42}$ was then used to determine the triplet quantum yield of $6 \mathrm{MP}, \Phi_{T}$, according to equation (6):

$$
\Phi_{T}=\frac{\Delta A_{6 M P}\left(\lambda_{4}\right) \Phi_{T}(R) \varepsilon_{R}^{*}\left(\lambda_{3}\right)}{\Delta A_{R}\left(\lambda_{3}\right) \varepsilon_{6 M P}^{*}\left(\lambda_{4}\right)}
$$

where $\Delta A_{6 M P}\left(\lambda_{4}\right)$ is the intensity of the triplet absorption of $6 \mathrm{MP}$ at $\lambda_{4}, \varepsilon_{6 M P}^{*}\left(\lambda_{4}\right)$ is the triplettriplet extinction coefficient of $6 \mathrm{MP}$ at $\lambda_{4}$, and $\Phi_{T}(R), \varepsilon_{R}^{*}\left(\lambda_{3}\right), \Delta A_{R}\left(\lambda_{3}\right)$ are the triplet quantum yield, the triplet-triplet extinction coefficient at $\lambda_{3}$, and the intensity of the triplet absorption at $\lambda_{3}$ for a reference compound of known triplet yield, respectively. In this case, we used 6thioguanosine as the reference compound, which has a reported triplet yield of $0.8 \pm 0.2$ in aqueous buffer solution. ${ }^{13} \lambda_{3}$ and $\lambda_{4}$ can be different wavelengths as long as they are wellseparated from the ground state bleaching signal. 
Singlet Oxygen Quantum Yields. Nanosecond time-resolved luminescence spectroscopy was used to determine the singlet oxygen quantum yields for 6MP and Aza following UVA excitation. Briefly, a GCR-150-30 Nd:YAG laser (Spectra Physics, 355 nm excitation wavelength, $7 \mathrm{~ns}$ pulse width) was used as the excitation source. Singlet oxygen phosphorescent decay traces were collected at $1270 \mathrm{~nm}$ using a modified Fluorolog-3 spectrometer (HORIBA, Jobin Yvon), with a near-IR sensitive photomultiplier tube (H10330A-45, Hamamatsu) as detector. The decay traces were stored on a digital oscilloscope (TDS 360, Tektronics). Solutions of 6MP, Aza, and the phenalenone standard were prepared in Tris-buffered $\mathrm{D}_{2} \mathrm{O}$ and acetonitrile at an optical density of 0.3 at $355 \mathrm{~nm}$ in $1 \mathrm{~cm}$ path length quartz cuvettes. The $\mathrm{O}_{2}$-saturated solutions were bubbled with ultrapure oxygen gas for at least 20 min prior to testing. Degradation of the samples was determined to be less than $3 \%$ over the course of the experiments based on their steady-state absorption spectra. The quantum yields were determined in back-to-back luminescence experiments of 6MP, Aza and phenalenone solutions under the same conditions, using the reported yield of ${ }^{1} \mathrm{O}_{2}$ generated by phenalenone as a standard $\left(\phi_{\Delta}=\right.$ $0.98) \cdot{ }^{43}$ 


\section{RESULTS}

\subsection{Steady-State Absorption and Emission of 6MP and Aza}
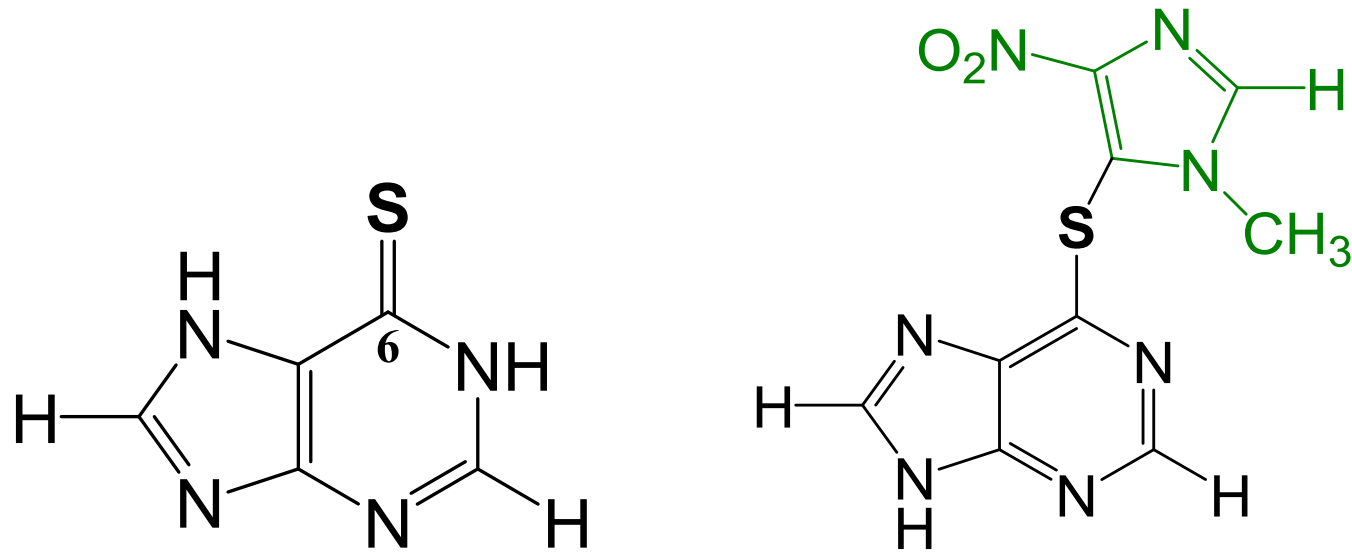

Scheme 1. Molecular structure of most stable tautomers of the neutral species of 6MP and Aza according to the electronic-structure calculations reported in this study in water.

6MP consists of a purine core chromophore thionated at the C6 position (Scheme 1). Aza has the same thiopurine chromophore but is functionalized with an electron-withdrawing nitroimidazole group at the sulfur atom (Scheme 1). The $\mathrm{pK}_{\mathrm{a}}$ corresponding to the purine core for these prodrugs lies between 7.7-7.87, ${ }^{44-47}$ which results in both $6 \mathrm{MP}$ and Aza existing as a mixture of the neutral and the conjugate base (deprotonated) species at physiological $\mathrm{pH}$. Using the Henderson-Hasselbalch relationship, we estimate that about $67 \%$ to $75 \%$ of the prodrugs exist in its neutral form at $\mathrm{pH} 7.4$, depending on the $\mathrm{pK}_{\mathrm{a}}$ value used, ${ }^{44-47}$ while the remaining $25 \%$ to $33 \%$ exist in the deprotonated form. Hence, to isolate the neutral and deprotonated species of 6MP and Aza, experiments were also performed in PBS at pH 5.5 (> 99\% neutral) and in borate buffer at $\mathrm{pH} 9.8$ (>99\% deprotonated).

Figure 1 shows the molar absorption spectra of 6MP and Aza in PBS solution at $\mathrm{pH} 5.5$, $\mathrm{pH} 7.4$, and in borate buffer at $\mathrm{pH}$ 9.8. The lowest-energy absorption band for the neutral form of $6 \mathrm{MP}$ has a maximum at $323 \mathrm{~nm}$, with a large molar absorption coefficient $\left(\varepsilon_{323}=22.7 \pm 0.3 \mathrm{mM}^{-}\right.$ 
${ }^{1} \mathrm{~cm}^{-1}$ in PBS at $\left.\mathrm{pH} 5.5\right)$. The absorption of 6MP does not change significantly in going from $\mathrm{pH}$ 5.5 to $\mathrm{pH}$ 7.4. However, there is a $5 \mathrm{~nm}$ blue shift in the absorption maximum $\left(\varepsilon_{318}=19.7 \pm 0.5\right.$ $\mathrm{mM}^{-1} \mathrm{~cm}^{-1}$ in PBS at $\mathrm{pH}$ 7.4). The deprotonated species of 6MP exhibits a further $5 \mathrm{~nm}$ blueshift in going from $\mathrm{pH} 7.4$ to $\mathrm{pH} 9.8$, which is accompanied by a decrease in molar absorption coefficient $\left(\varepsilon_{313}=12.6 \pm 0.3 \mathrm{mM}^{-1} \mathrm{~cm}^{-1}\right.$ in borate buffer at $\left.\mathrm{pH} 9.8\right)$.

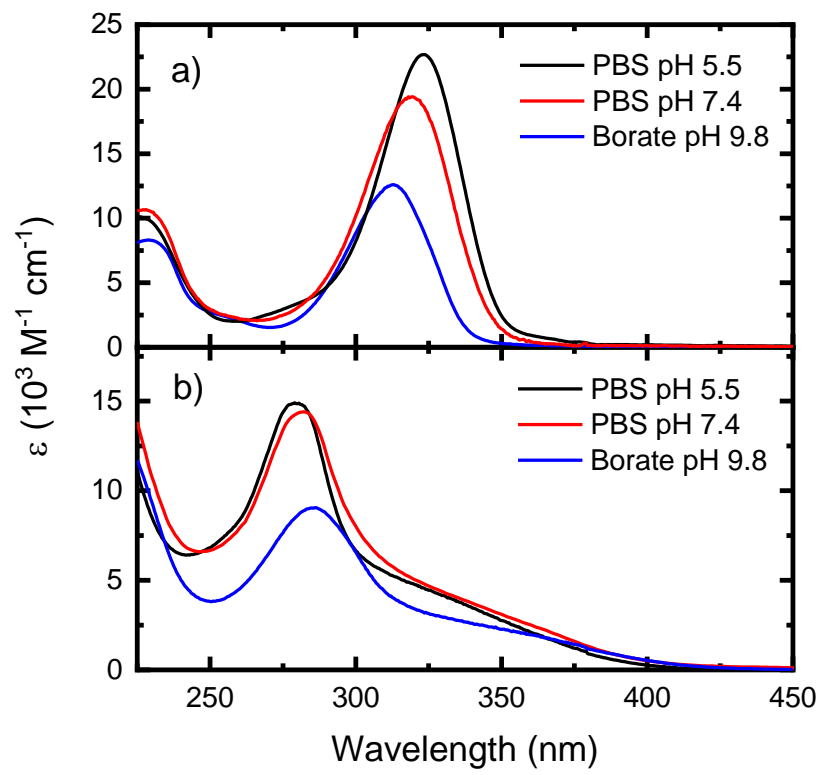

Figure 1. Molar absorption spectra of a) $6 \mathrm{MP}$ and b) Aza as a function of $\mathrm{pH}$.

Figure 1b shows the molar absorption spectra of Aza in PBS at pH 5.5 and 7.4, and in borate buffer at $\mathrm{pH}$ 9.8. The nitroimidazole functionalization blueshifts the absorption maximum by $44 \mathrm{~nm}$ to $279 \mathrm{~nm}$ relative to the $6 \mathrm{MP}$ neutral species $\left(\varepsilon_{279}=14.8 \pm 0.4 \mathrm{mM}^{-1} \mathrm{~cm}^{-1}\right.$ in $\mathrm{PBS}$ at $\mathrm{pH}$ 5.5), while simultaneously broadening the spectrum into the near visible (Figure 1b). The absorption of the neutral and the deprotonated mixture at $\mathrm{pH} 7.4$ is $2 \mathrm{~nm}$ redshifted but essentially of the same intensity as that of the neutral form of Aza $\left(\varepsilon_{281}=14.4 \pm 0.2 \mathrm{mM}^{-1} \mathrm{~cm}^{-1}\right.$ in 
PBS at $\mathrm{pH}$ 7.4). An additional $4 \mathrm{~nm}$ redshift and a slight decrease in molar absorption is observed for the deprotonated form of Aza $\left(\varepsilon_{285}=11.7 \pm 0.3 \mathrm{mM}^{-1} \mathrm{~cm}^{-1}\right.$ in borate buffer at $\mathrm{pH}$ 9.8).

Figures $\mathrm{S} 1$ to $\mathrm{S} 3$ show that low-intensity emission bands are observed for 6MP and Aza following excitation at $334 \mathrm{~nm}$ in the $\mathrm{pH}$ range from 5.5 to 9.8. The primary emission band for 6MP at $495 \mathrm{~nm}$ is assigned to phosphorescence emission because it exhibits a large Stokes' shift relative to the lowest-energy absorption band and can be quenched by $\mathrm{O}_{2}$. Another emission band is observed at ca. $370 \mathrm{~nm}$, which is assigned to fluorescence emission because it exhibits a relatively small Stokes's shift and is not quenched by $\mathrm{O}_{2}$. The excitation spectra reported in Figures $\mathrm{S} 1$ to $\mathrm{S} 3$ evidence that the emission is an intrinsic property of the 6MP prodrug and not due to impurities. Fluorescence and phosphorescence quantum yields as a function of $\mathrm{pH}$ were not determined because the emission signals are within the detection limit of the spectrometer. However, we can estimate quantum yields in the order of $10^{-4}$, as has been observed for the other thiobases. ${ }^{18,21}$

Aza also exhibits a small-intensity fluorescence emission band in PBS at pH 5.5 and 7.4, and in borate buffer at $\mathrm{pH} 9.8$ at $425 \mathrm{~nm}$ (Figures $\mathrm{S} 1$ to S3). The emission band features a small Stokes shift relative to the lowest-energy absorption shoulder and is not quenched by $\mathrm{O}_{2}$. As for $6 \mathrm{MP}$, fluorescence quantum yields and excitation spectra as a function of $\mathrm{pH}$ were not obtained because the emission signal is within the detection limits of the spectrometer.

\subsection{Theoretical Calculations}

\subsubsection{Acid-Base and Prototropic Tautomerization of Aza and 6MP}

As mentioned above, the $\mathrm{pK}_{\mathrm{a}}$ corresponding to the thiopurine core for these molecules lies between 7.7-7.87, ${ }^{44-47}$ which makes both 6MP and Aza exist as a mixture of neutral (67- 
$75 \%$ ) and deprotonated (25-33\%) species at physiological $\mathrm{pH}$. Moreover, prototropic tautomerism is well-known to exist in purine derivatives. ${ }^{48-50}$ Previous work ${ }^{51}$ on the prototropic tautomerization of neutral Aza in aqueous solution has shown that neutral Aza exists only as the $\mathrm{N} 9(\mathrm{H})$ tautomer ${ }^{51}$ while the deprotonated form of Aza cannot exhibit prototropic tautomerization. In the case $6 \mathrm{MP}$, the prototropic tautomerization of the neutral species have been studied in deuterated-dimethylsulfoxide, deuterated-dimethylformamide, and deuteratedmethanol. The investigation demonstrated that the $\mathrm{N} 7(\mathrm{H})-\mathrm{N} 1(\mathrm{H})$ is the predominant species of 6MP in these solvents. ${ }^{49,52}$ In order to determine which tautomer(s) is available in aqueous solution, the ground-state geometries of four tautomers of the neutral form of 6MP (i.e., N7(H)$\mathrm{N} 1(\mathrm{H}), \mathrm{N} 9(\mathrm{H})-\mathrm{N} 1(\mathrm{H}), \mathrm{N} 7(\mathrm{H})-\mathrm{N} 3(\mathrm{H})$, and $\mathrm{N} 9(\mathrm{H})-\mathrm{N} 3(\mathrm{H}))$ were optimized in this study at the DFT-B3LYP/6-311+G(d,p) level of theory including both implicit (IEFPCM = water) solvent effects and two explicit water molecules to form hydrogen bonds with the nitrogen atoms of the thiopurine core. Single-point calculations at the MP2 level of theory where then used on the optimized geometries to calculate the relative energies among tautomers. The relative energies predict that the $\mathrm{N} 7(\mathrm{H})-\mathrm{N} 1(\mathrm{H})$ tautomer is $8.4,52.7$, and $55.2 \mathrm{~kJ} / \mathrm{mol}$ more stable than the $\mathrm{N} 9(\mathrm{H})$ $\mathrm{N} 1(\mathrm{H}), \mathrm{N} 7(\mathrm{H})-\mathrm{N} 3(\mathrm{H})$, and $\mathrm{N} 9(\mathrm{H})-\mathrm{N} 3(\mathrm{H})$ tautomers in aqueous solution, respectively, at the MP2 level of theory. The same tautomeric order was predicted at DFT level of theory. Using this methodology but adding three water molecules to form hydrogen bonds with the available nitrogen atoms, it was determined that the $\mathrm{N} 1(\mathrm{H})$ tautomer of the deprotonated form of $6 \mathrm{MP}$ is $6.3,18.8,36.8 \mathrm{~kJ} / \mathrm{mol}$ more stable than the $\mathrm{N} 7(\mathrm{H}), \mathrm{N} 9(\mathrm{H})$, and $\mathrm{N} 3(\mathrm{H})$, respectively, at the MP2 level of theory. The same tautomeric order was predicted at the DFT level of theory. Considering that the relative energy between the most stable and the second most stable tautomer in both the neutral and deprotonated forms is well-above $k T$ at room temperature, the calculations predict 
that the $\mathrm{N} 7(\mathrm{H})-\mathrm{N} 1(\mathrm{H})$ and the $\mathrm{N} 1(\mathrm{H})$ tautomers are the only available species in solution for the neutral and deprotonated forms, respectively. Therefore, all the calculations presented hereafter were performed only for the $\mathrm{N} 9(\mathrm{H})$ tautomer of neutral Aza and for the N7(H)-N1(H) tautomer of the neutral and the $\mathrm{N} 1(\mathrm{H})$ tautomer of the deprotonated forms of 6MP. Microsolvation for the neutral and the deprotonated form of Aza was modeled with four and five water molecules, respectively. The water molecules were added to form hydrogen bonds with the available nitrogen atoms. The predicted acid-base equilibria for 6MP and Aza at $\mathrm{pH} 7.4$ are shown in Scheme 2, together with the ground-state optimized geometries of the lowest-energy available tautomer in solution. Note that upon optimization of the deprotonated form of Aza, a water molecule that was added to form a hydrogen bond with the $\mathrm{N} 3$ atom migrated to form hydrogen bond with one of the oxygens of the nitroimidazole (Scheme 2).
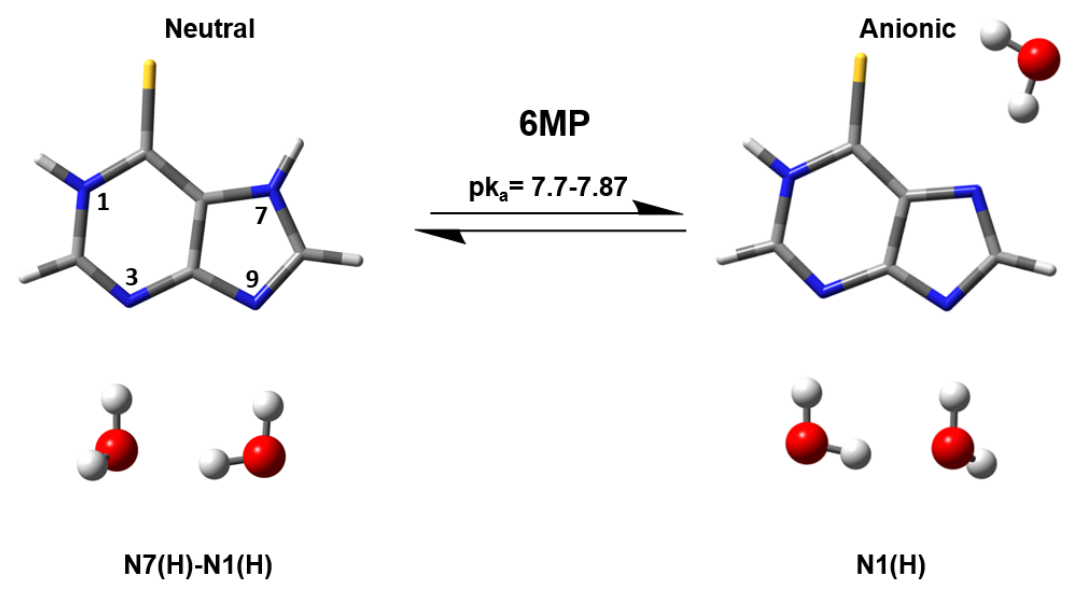

$67-75 \%$

N1(H)

$25-33 \%$ 


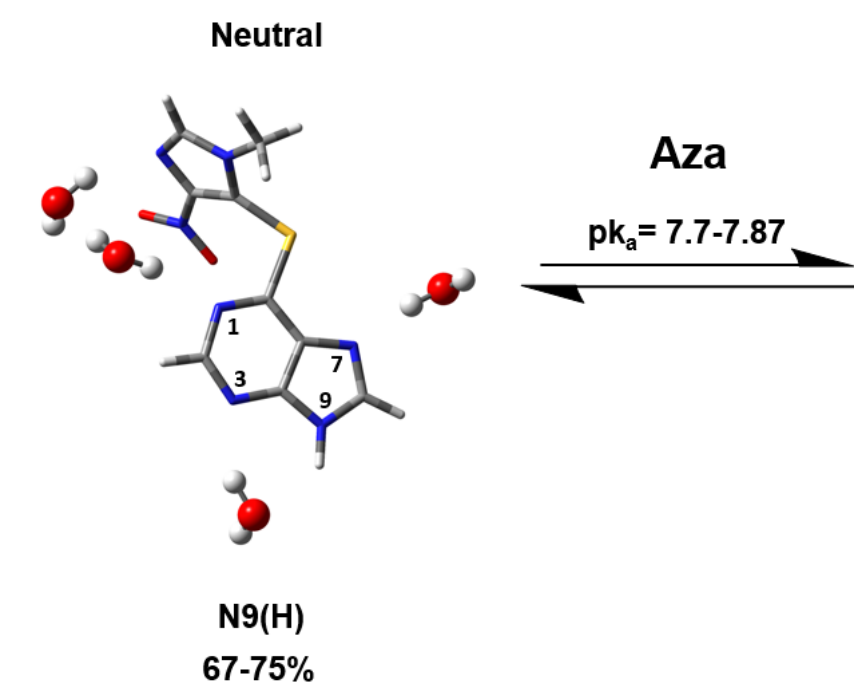

Anionic

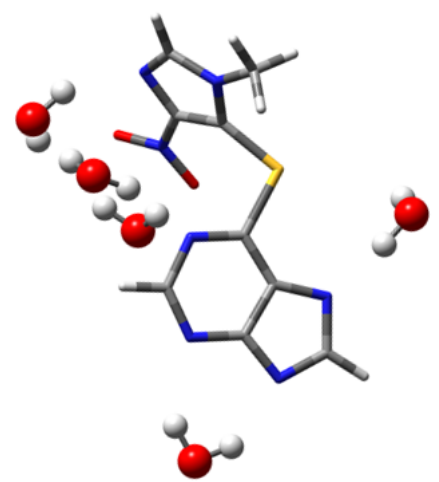

$25-33 \%$

Scheme 2. Proposed acid-base equilibrium of 6MP (top) and Aza (bottom). Tautomers shown are the microsolvated ground-state optimized structures for the tautomers available in aqueous solution at $\mathrm{pH}$ 7.4. All optimizations were performed at the B3LYP/IEFPCM/6-311+G(d,p) level of theory in water.

\subsubsection{Vertical Excitation Energies and Spin-Orbit Coupling Constants}

In order to characterize the electronic states available upon UVA excitation of 6MP, VEEs were calculated at the TD-X/IEFPCM/6-311+G(d,p) level of theory using the optimized neutral and deprotonated micro-solvated structures in water, where $\mathrm{X}$ is the functional used. In this study, we used the PBE0, $\omega \mathrm{B} 97 \mathrm{X}$ and CAM-B3LYP functionals for comparison. The $\mathrm{S}_{2}$ state is predicted to be directly populated upon UVA excitation of both the neutral and the deprotonated forms of 6MP. This state has $\pi \pi^{*}$ character with an oscillator strength of $\sim 0.5$ in both the neutral and the deprotonated forms of 6MP (Figure 2 and Table 1). Below the $S_{2}\left(\pi \pi^{*}\right)$ state, there are four excited electronic states available for deactivation (i.e., $S_{1}, T_{3}, T_{2}$ and $T_{1}$ states). The $S_{1}$ state has $n \pi^{*}$ character with a negligible oscillator strength of 0.000 , while the $T_{3}$, $\mathrm{T}_{2}$ and $\mathrm{T}_{1}$ states have $\pi \pi^{*}$, n $\pi^{*}$ and $\pi \pi^{*}$ characters, respectively. The SOCs between the two lowest-energy singlet states and three lowest triplet states were calculated using the FranckCondon geometry and are reported in Table 2 . As shown in Table 2, the $S_{2} \rightarrow T_{2}$ and $S_{1} \rightarrow T_{1}$ 
are the only two transitions that exhibit SOCs of significantly large magnitude $\left(\sim 120 \mathrm{~cm}^{-1}\right)$, independent of the functional used, and are thus expected to be involved in the intersystem crossing pathway to the triplet manifold. The magnitude of the SOCs is also in agreement with El-Sayed's propensity rules.

Therefore, based in these calculations, it is predicted that excitation at $334 \mathrm{~nm}$ of both the neutral and the deprotonated forms of $6 \mathrm{MP}$ leads to the population of the $\mathrm{S}_{2}\left(\pi \pi^{*}\right)$ state in aqueous solution, which can rapidly intersystem cross to the triplet manifold by two competing pathways. The first pathway is direct intersystem crossing from the $S_{2}\left(\pi \pi^{*}\right)$ state to the $T_{2}\left(n \pi^{*}\right)$ state. The second pathway involves internal conversion from the $S_{2}\left(\pi \pi^{*}\right)$ state to the $S_{1}\left(n \pi^{*}\right)$ state, followed by intersystem crossing to the $T_{1}\left(\pi \pi^{*}\right)$ state. Because the $S_{2}\left(\pi \pi^{*}\right), S_{1}\left(n \pi^{*}\right)$, $\mathrm{T}_{2}\left(\mathrm{n} \pi^{*}\right)$ and $\mathrm{T}_{1}\left(\pi \pi^{*}\right)$ states are all predicted to play a relevant role in the electronic relaxation of $6 \mathrm{MP}$, the nuclear coordinates of the four electronic states were also optimized at the TDPBE0/IEFPCM/6-311+G(d,p) level of theory in water and their excited state absorption spectra were calculated at the TD-PBE0/CPCM/6-311+G(d,p) in the same solvent.

The optimized structures and excited state absorption spectra for all electronic excited states are reported in Figure $S 4$ and S5, respectively. In the $S_{2}\left(\pi \pi^{*}\right)$ and the $S_{1}\left(n \pi^{*}\right)$ minima, the purine chromophore remains planar. However, in the case of the $S_{2}\left(\pi \pi^{*}\right)$ minimum the sulfur atom is out of the molecular plane of the purine chromophore, while the sulfur remains in the molecular plane of the purine chromophore in the $S_{1}\left(n \pi^{*}\right)$ minimum. Similarly, the purine chromophore remains planar in the case of the $\mathrm{T}_{1}\left(\pi \pi^{*}\right)$ and $\mathrm{T}_{2}\left(\mathrm{n} \pi^{*}\right)$ optimized geometries, but the sulfur atom is out of the plane of the purine chromophore in the $T_{2}\left(n \pi^{*}\right)$ minimum, while the sulfur atom remains in the plane of the chromophore in the $\mathrm{T}_{1}\left(\pi \pi^{*}\right)$ minimum at the TDPBE0/IEFPCM/6-311+G(d,p) level of theory. 
The $\mathrm{T}_{1}\left(\pi \pi^{*}\right)$ state was also optimized with DFT at the UPBE0/IEFPCM/6-311+G(d,p) level of theory in water, and its excited state absorption spectrum was calculated at the TDPBE0/IEFPCM/6-311+G(d,p) level of theory. Importantly, as noted above, optimization of the $\mathrm{T}_{1}\left(\pi \pi^{*}\right)$ state at the TD-PBE0/IEFPCM/6-311+G(d,p) level of theory yielded a planar minima (Figure S4), whereas the optimization of the $\mathrm{T}_{1}\left(\pi \pi^{*}\right)$ state at the UPBE0/IEFPCM/6-311+G(d,p) level of theory yielded an out-of-plane minimum (Figure S4), in which the sulfur atom is out of the molecular plane. We note that previous multiconfigurational calculations have demonstrate the presence of two triplet minima in the $\mathrm{T}_{1}\left(\pi \pi^{*}\right)$ potential energy surface of several the thiobases, ${ }^{53-55}$ which has been supported by experimentation. ${ }^{55,56}$ Collectively, the computational and experimental results suggest that the topology of the $T_{1}\left(\pi \pi^{*}\right)$ potential energy surface is characterized by the presence of at least two minima, ${ }^{53}$ which can play an important role in the generation of reactive oxygen species (see discussion section).

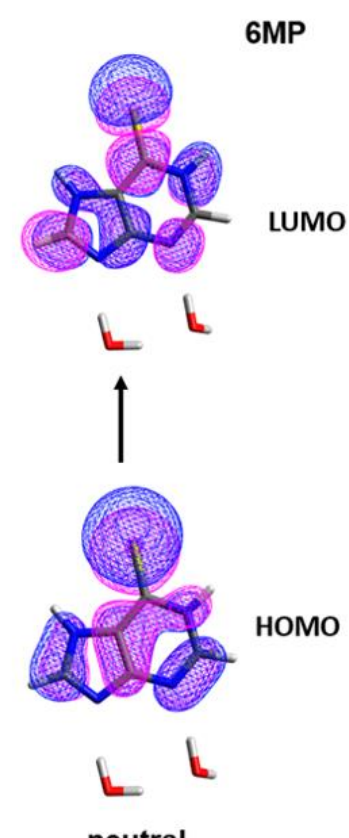

neutral
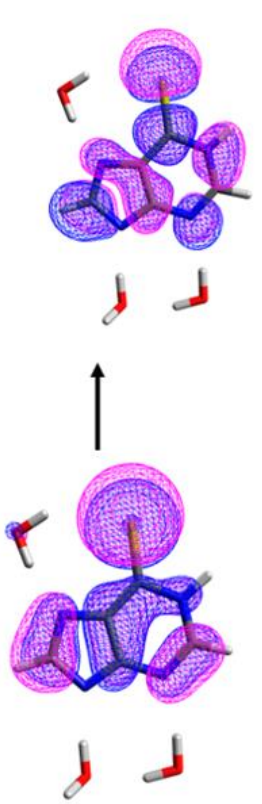

anionic

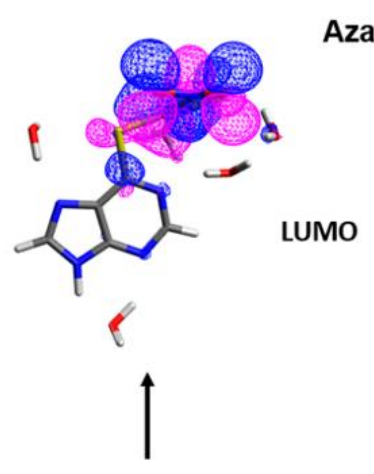

Aza
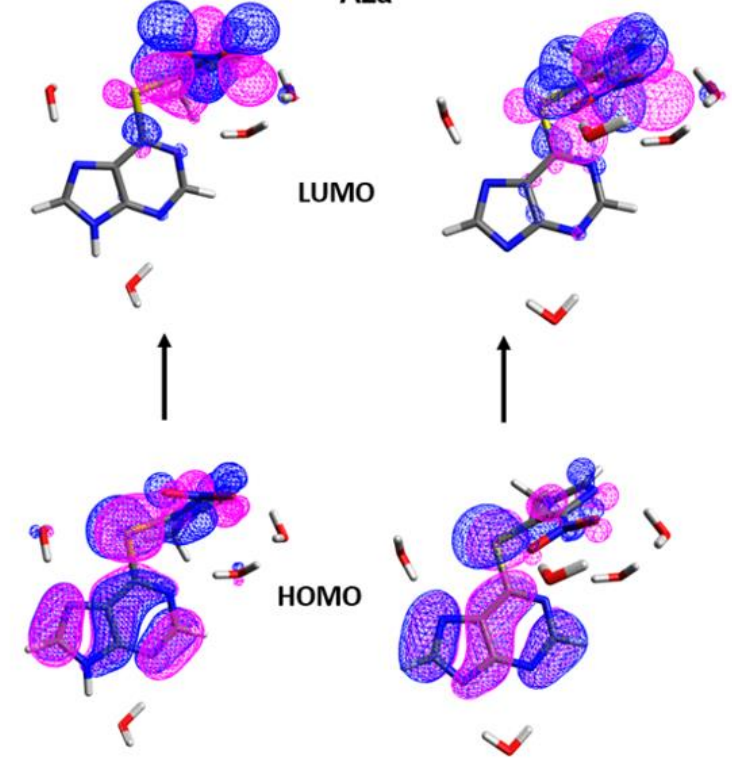

neutral

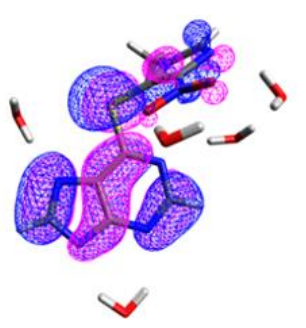

anionic

Figure 2. HOMO $\rightarrow$ LUMO single electron transitions for 6MP (left) and Aza (right) calculated at the TD$\mathrm{X} / \mathrm{IEF}-\mathrm{PCM} / 6-311+\mathrm{G}(\mathrm{d}, \mathrm{p})$ level of theory in water, where $\mathrm{X}$ is the functional used $(\mathrm{X}=\mathrm{PBE} 0$ for $6 \mathrm{MP}$ and 
$\mathrm{X}=\omega \mathrm{B} 97 \mathrm{X}$ for Aza). In neutral and deprotonated $6 \mathrm{MP}$, the $\mathrm{HOMO} \rightarrow \mathrm{LUMO}$ single electron transition accounts for $>95 \%$ of the $\pi \pi^{*}$ character of the $\mathrm{S}_{2}$ state. In neutral and deprotonated Aza the $\mathrm{HOMO} \rightarrow$ LUMO single electron transition accounts for $>41 \%$ of the $\pi \pi^{*}(\mathrm{CT})$ character of the $\mathrm{S}_{2}$ state and $>18 \%$ of the $\mathrm{S}_{1}$ state.

Table 1. Vertical excitation energies (in $\mathrm{eV}$ ) and relevant energy gaps of micro-solvated 6MP calculated at the TD-X/IEF-PCM/6-311+G(d,p) level of theory in water, where $\mathrm{X}$ is the functional used ${ }^{\mathrm{a}}$

\begin{tabular}{lllllll}
\hline State & \multicolumn{3}{c}{ Neutral } & \multicolumn{3}{c}{ Deprotonated } \\
\hline & CAM-B3LYP & $\omega$ B97X & PBE0 & CAM-B3LYP & $\omega B 97 X$ & PBE0 \\
$\mathrm{S}_{2}\left(\pi \pi^{*}\right)$ & $3.92(0.444)$ & $4.00(0.486)$ & $3.84(0.367)$ & $4.15(0.503)$ & $4.21(0.523)$ & $4.13(0.470)$ \\
$\mathrm{S}_{1}\left(\mathrm{n} \pi^{*}\right)$ & $3.54(0.000)$ & $3.74(0.000)$ & $3.32(0.000)$ & $3.83(0.000)$ & $4.01(0.000)$ & $3.67(0.000)$ \\
$\mathrm{T}_{3}\left(\pi \pi^{*}\right)$ & 3.49 & 3.59 & 3.42 & 3.80 & 3.94 & 3.67 \\
$\mathrm{~T}_{2}\left(\mathrm{n} \pi^{*}\right)$ & 3.30 & 3.52 & 3.08 & 3.61 & 3.81 & 3.44 \\
$\mathrm{~T}_{1}\left(\pi \pi^{*}\right)$ & 2.43 & 2.48 & 2.44 & 2.77 & 2.82 & 2.78 \\
$\Delta \mathrm{E}\left(\mathrm{S}_{2}-\mathrm{S}_{1}\right)$ & 0.4 & 0.3 & 0.5 & 0.3 & 0.2 & 0.5 \\
$\Delta \mathrm{E}\left(\mathrm{S}_{2}-\mathrm{S}_{2}\right)$ & 0.6 & 0.5 & 0.8 & 0.5 & 0.4 & 0.7 \\
$\Delta \mathrm{E}\left(\mathrm{S}_{1}-\mathrm{T}_{1}\right)$ & 1.1 & 1.3 & 0.9 & 1.1 & 1.2 & 0.9
\end{tabular}

${ }^{\text {a }}$ Oscillator strengths are shown in parentheses. Energy gaps were rounded to one decimal place.

Table 2. Spin-orbit coupling constants $\left(\right.$ in $\mathrm{cm}^{-1}$ ) between relevant singlet and triplet states of microsolvated 6MP calculated at the TD-X/CPCM/6-311+G(d,p) level of theory in water, where $\mathrm{X}$ is the functional used

\begin{tabular}{lllllll}
\hline Transition & \multicolumn{3}{c}{ Neutral } & \multicolumn{3}{c}{ Deprotonated } \\
\hline & CAM-B3LYP & $\omega B 97 X$ & PBE0 & CAM-B3LYP & $\omega B 97 X$ & PBE0 \\
$\mathrm{S}_{1}-\mathrm{T}_{2}$ & 0.08 & 0.07 & 0.16 & 0.31 & 0.33 & 0.33 \\
$\mathrm{~S}_{1}-\mathrm{T}_{1}$ & 126 & 123 & 127 & 112 & 107 & 113 \\
$\mathrm{~S}_{2}-\mathrm{T}_{1}$ & 0.04 & 0.04 & 0.10 & 0.01 & 0.02 & 0.02 \\
$\mathrm{~S}_{2}-\mathrm{T}_{2}$ & 124 & 123 & 113 & 121 & 119 & 114 \\
$\mathrm{~S}_{2}-\mathrm{T}_{3}$ & 0.07 & 0.07 & 0.11 & 0.07 & 0.08 & 0.07
\end{tabular}

Vertical excitation energies were also calculated for the neutral and deprotonated forms of Aza using the CAM-B3LYP, $\omega$ B97X, and the PBE0 functionals. The calculations show that the PBE0 and the CAM-B3LYP functionals underperformed relative to the $\omega \mathrm{B} 97 \mathrm{X}$ functional (Figure S6). Therefore, the results obtained with the PBE0 and the CAM-B3LYP functionals are not discussed further. The VEEs for the two lowest-energy excited singlets states and five/six lowest-energy excited triplet states for the neutral/deprotonated form of Aza are reported in Table 3. Excitation of the neutral form of Aza at 334 or $316 \mathrm{~nm}$ results in the simultaneous population of the $S_{1}$ and $S_{2}$ states (see Figure $S 7$, for details). The $S_{1}$ state exhibits a mixture of 
$\pi \pi^{*}$ and $\mathrm{n} \pi^{*}$ character $(>52 \%)$ and significant $(42 \%) \pi \pi^{*}$ charge transfer (CT) character, regardless of the protonation status (i.e., electron density moves from the purine chromophore to the nitroimidazole group, see Figure 2). The $S_{2}$ state has $\pi \pi^{*}$ character with significant (>73\%) CT character. Below the $S_{2}\left(\pi \pi^{*}, \mathrm{CT}\right)$ state, there are five and six excited triplet states available for deactivation for the neutral and deprotonated forms of Aza, respectively. For the neutral Aza, the $T_{1}, T_{4}$ and $T_{5}$ states exhibit a mixture of $\pi \pi^{*}$ and $n \pi^{*}$ characters, while the $T_{2}$ state has both $\pi \pi^{*}$ and CT characters. The $\mathrm{T}_{3}$ state has purely $\pi \pi^{*}$ character. For the deprotonated form, the $\mathrm{T}_{1}$ and $\mathrm{T}_{4}$ states exhibit a mixture of $\pi \pi^{*}$ and $n \pi^{*}$ characters, while the $\mathrm{T}_{2}$ and $\mathrm{T}_{6}$ states have both $\pi \pi^{*}$ and $\mathrm{CT}$ characters. The $\mathrm{T}_{3}$ and $\mathrm{T}_{5}$ states have pure $\pi \pi^{*}$ character.

Table 3. Vertical excitation energies (in eV) and relevant energy gaps of micro-solvated neutral and deprotonated Aza calculated at the TD- $\omega$ B97X /IEFPCM/6-31+G(d,p) level of theory in water

\begin{tabular}{lll}
\hline State & \multicolumn{1}{c}{ Neutral } & \multicolumn{1}{c}{ Deprotonated } \\
\hline $\mathrm{S}_{1}$ & $3.87(0.019)$ & $3.85(0.038)$ \\
& $52 \% \pi \pi^{*}+\mathrm{n} \pi^{*}$ & $58 \% \pi \pi^{*}+\mathrm{n} \pi^{*}$ \\
& $48 \% \pi \pi^{*}, \mathrm{CT}$ & $42 \% \pi \pi^{*}, \mathrm{CT}$ \\
$\mathrm{S}_{2}$ & $4.05(0.152)$ & $4.16(0.113)$ \\
& $73 \% \pi \pi^{*}, \mathrm{CT}$ & $74 \% \pi \pi^{*}, \mathrm{CT}$ \\
$\mathrm{T}_{1}$ & 2.78 & 2.82 \\
& $76 \% \pi \pi^{*}+\mathrm{n} \pi^{*}$ & $70 \% \pi \pi^{*}+\mathrm{n} \pi^{*}$ \\
$\mathrm{~T}_{2}$ & 3.13 & 3.10 \\
& $96 \% \pi \pi^{*}, \mathrm{CT}$ & $81 \% \pi \pi^{*}, \mathrm{CT}$ \\
$\mathrm{T}_{3}$ & 3.43 & 3.39 \\
& $69 \% \pi \pi^{*}$ & $95 \% \pi \pi^{*}$ \\
$\mathrm{~T}_{4}$ & 3.44 & 3.45 \\
& $65 \% \pi \pi^{*}+\mathrm{n} \pi^{*}$ & $83 \% \pi \pi^{*}+\mathrm{n} \pi^{*}$ \\
$\mathrm{~T}_{5}$ & 4.09 & 4.03 \\
& $80 \% \pi \pi^{*}+\mathrm{n} \pi *$ & $95 \% \pi \pi^{*}$ \\
$\Delta \mathrm{E}\left(\mathrm{S}_{2}-\mathrm{S}_{1}\right)$ & 0.2 & 0.4 \\
$\Delta \mathrm{E}\left(\mathrm{S}_{1}-\mathrm{T}_{1}\right)$ & 1.0 & 0.8 \\
$\Delta \mathrm{E}\left(\mathrm{S}_{1}-\mathrm{T}_{2}\right)$ & 0.7 & 0.7 \\
$\Delta \mathrm{E}\left(\mathrm{S}_{2}-\mathrm{T}_{2}\right)$ & 0.9 & 1.1
\end{tabular}

${ }^{a}$ Oscillator strengths are shown in parentheses. In the case of deprotonated Aza, $\mathrm{T}_{6}\left(4.09,86 \% \pi \pi^{*}, \mathrm{CT}\right)$ is also lower in energy than the $S_{2} . \pi \pi^{*}+n \pi^{*}$ corresponds to transitions where the HOMO-X in the single electron transition has both $\mathrm{n}$ orbital (in the nitroimidazole) and $\pi$ orbital (in the purine) characters. 
Table 4 reports the SOCs between relevant singlet and triplet states. The $S_{1} \rightarrow T_{2}\left(43 \mathrm{~cm}^{-}\right.$ $\left.{ }^{1}\right), S_{2} \rightarrow T_{2}\left(20 \mathrm{~cm}^{-1}\right), S_{2} \rightarrow T_{1}\left(22 \mathrm{~cm}^{-1}\right)$, and $S_{2} \rightarrow T_{4}\left(21 \mathrm{~cm}^{-1}\right)$ electronic transitions exhibit the largest SOCs for the neutral of Aza. For the deprotonated form, the $S_{1} \rightarrow T_{2}\left(27 \mathrm{~cm}^{-1}\right)$ and $S_{2} \rightarrow$ $\mathrm{T}_{2}\left(37 \mathrm{~cm}^{-1}\right)$ transitions exhibit the largest SOCs. As shown in Tables 2 and 4 , the magnitudes of SOCs are significantly reduced compared to 6MP, but they are still relatively large, i.e., in the order of tens of wavenumbers. Due to the molecular size of the neutral and deprotonated forms, we were unable to optimize the electronic excited states of Aza within the computational resources available to us.

Table 4. Spin-orbit coupling constants (in $\mathrm{cm}^{-1}$ ) between relevant singlet and triplet states of microsolvated Aza calculated at the TD- $\omega$ B97X /CPCM/6-31+G(d,p) level of theory in water

\begin{tabular}{lll}
\hline Transition & \multicolumn{1}{c}{ Neutral } & Deprotonated \\
\hline $\mathrm{S}_{1}-\mathrm{T}_{1}$ & 7.3 & 14 \\
$\mathrm{~S}_{1}-\mathrm{T}_{2}$ & 43 & 27 \\
$\mathrm{~S}_{1}-\mathrm{T}_{3}$ & 1.6 & 10 \\
$\mathrm{~S}_{1}-\mathrm{T}_{4}$ & 10 & 3.3 \\
$\mathrm{~S}_{2}-\mathrm{T}_{1}$ & 22 & 11 \\
$\mathrm{~S}_{2}-\mathrm{T}_{2}$ & 20 & 37 \\
$\mathrm{~S}_{2}-\mathrm{T}_{3}$ & 5.2 & 12 \\
$\mathrm{~S}_{2}-\mathrm{T}_{4}$ & 21 & 5.0 \\
$\mathrm{~S}_{2}-\mathrm{T}_{5}$ & 3.2 & 3.0 \\
$\mathrm{~S}_{2}-\mathrm{T}_{6}$ & - & 7.0
\end{tabular}

\subsubsection{Calculation of Thermodynamic values for 6MP}

Molecular oxygen can quench the triplet state of 6MP forming reactive oxygen species other than ${ }^{1} \mathrm{O}_{2}$. For instance, electron transfer between the triplet state of $6 \mathrm{MP}$ and $\mathrm{O}_{2}$ can give rise to the superoxide radical, as previously proposed for 6-thioguanosine and 6-thioguanine. ${ }^{16,17}$ Hence, the oxidation potential and adiabatic triplet energy for the neutral and the deprotonated forms of $6 \mathrm{MP}$ were calculated to estimate the Gibbs free energy associated with the electron transfer process $\left(\Delta \mathrm{G}_{\mathrm{ET}}\right)$ using the Rehm-Weller equation (see Methodology for details). ${ }^{33}$ The 
results from these calculations are reported in Table 5 using both the planar and out-of-plane $\mathrm{T}_{1}$ minima calculated in this study.

Table 5. Calculated values (in $\mathrm{kJ} / \mathrm{mol}$ ) used to estimate the Gibbs free energy for electron transfer $\left(\Delta \mathrm{G}_{\mathrm{ET}}\right)$ between the lowest energy triplet state of neutral and deprotonated 6MP and the ground state molecular oxygen

\begin{tabular}{ccc}
\hline Energy & Neutral & Deprotonated \\
\hline $\mathrm{E}_{\mathrm{T}}^{\#}$ & $283.4^{\S \S}(241.2)^{\dagger \dagger}$ & $249.2^{\S \S}(234.1)^{\dagger \dagger}$ \\
$\mathrm{VIE}^{\dagger}$ & 821.3 & 325.4 \\
$\mathrm{E}_{\mathrm{ox}}^{\S}$ & 210.2 & -67.7 \\
$\Delta \mathrm{G}_{\mathrm{ET}}^{\dagger}$ & $-129.3^{\S \S}(-81.2)^{\dagger \dagger}$ & $-374.0^{\S \S}(-365.7)^{\dagger \dagger}$
\end{tabular}

\# Adiabatic energy of the lowest energy triplet state $\left(3 \pi \pi^{*}\right)$ of 6MP in water;

$\dagger$ vertical ionization energy of 6MP in vacuum at the B3LYP/6-31++G(d,p)//UB3LYP/6-31++G(d,p) level of theory;

$\S$ oxidation potential of $6 \mathrm{MP}$ was estimated using Eq. (4) in ref. $\left({ }^{30}\right)$, corrected for the zero-point-energy, and using the VIE calculated in this work;

$\S \S$ energy of the out-of-plane $\mathrm{T}_{1}$ minimum obtained using the UPBE0/IEFPCM/6-311+G(d,p) level of theory;

${ }^{\dagger}$ energy of the planar $\mathrm{T}_{1}$ minimum obtained using the TD-PBE0/IEFPCM/6-311+G(d,p) level of theory.

\subsection{Femtosecond Transient Absorption Experiments for 6MP and Aza}

\subsection{1. $6 \mathrm{MP}$}

Femtosecond broadband transient absorption experiments were performed to investigate the electronic relaxation pathways and to quantify their rates. Figure 3 presents the transient absorption spectra of 6MP upon excitation at $334 \mathrm{~nm}$. As can be seen in Figure 3a-c, at time delays up to time zero, a band grows with maxima in the range of 550 to $600 \mathrm{~nm}$ regardless of the $\mathrm{pH}$ used. After time zero, a transient signal develops exhibiting absorption maxima at ca. 480 and $690 \mathrm{~nm}$ at $\mathrm{pH} 5.5$ and 7.4, while the higher energy band is blue shifted to ca. $510 \mathrm{~nm}$ at $\mathrm{pH}$ 9.8. This transient species continues to slightly blueshift and its intensity to increase for up to $600 \mathrm{ps}$ in $\mathrm{pH} 5.5$ and 7.4, and for up to $900 \mathrm{ps}$ in $\mathrm{pH}$ 9.8. The transient species then remains 
practically constant thereafter for up to $3 \mathrm{~ns}$. Lifetimes and evolution-associated difference spectra (EADS) extracted from the global and target analyses are reported in Table 6 and Figure S8, respectively.
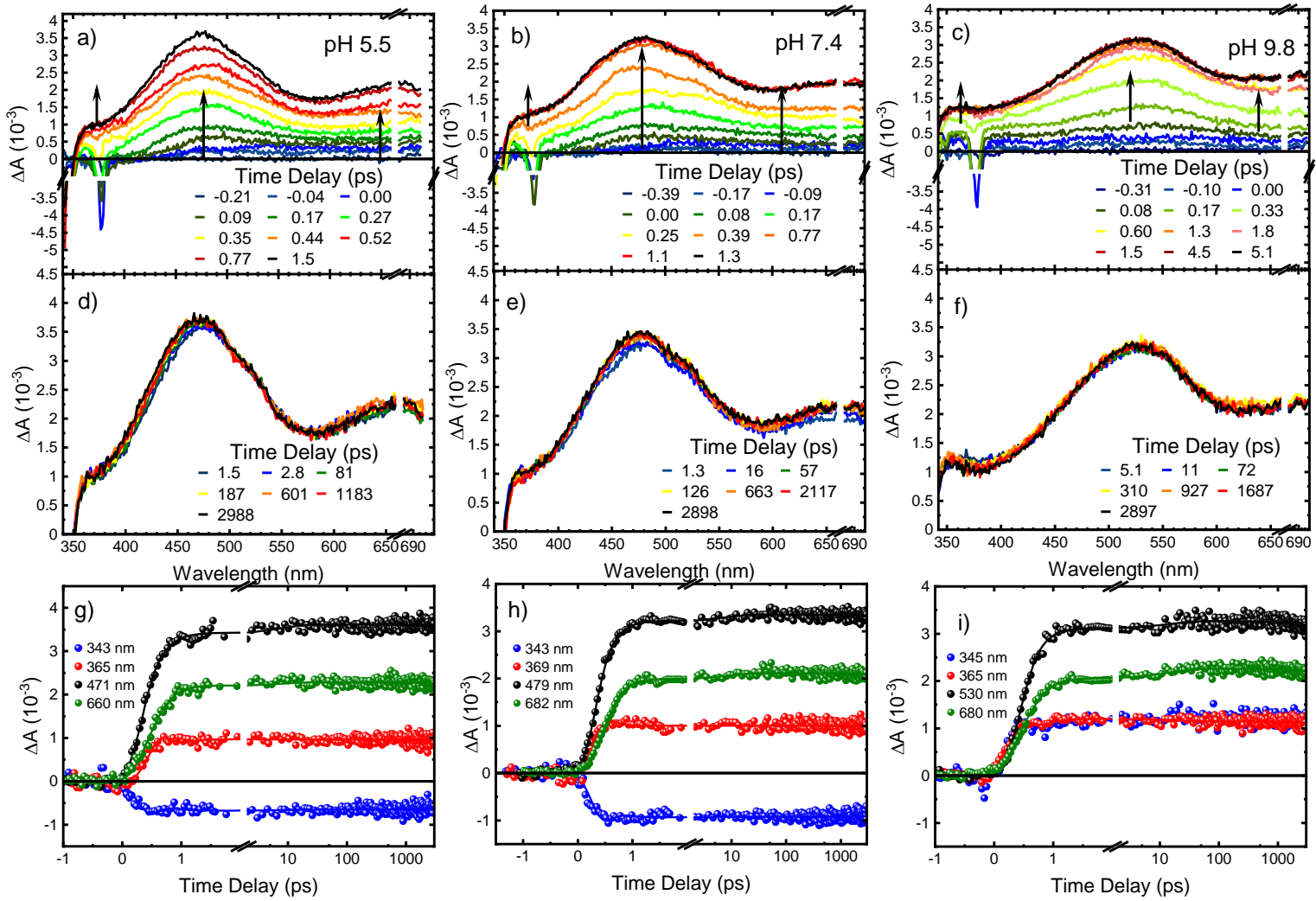

Figure 3. Transient absorption spectra for $6 \mathrm{MP}\left(\lambda_{\mathrm{exc}}=334 \mathrm{~nm}\right)$ in PBS pH 5.5 and $\mathrm{pH} 7.4$ and borate buffer $\mathrm{pH} 9.8$ (a-f). Representative decay traces in $\mathrm{g}$ ) $\mathrm{pH} 5.5, \mathrm{~h}$ ) $\mathrm{pH} 7.4$ and i) $\mathrm{pH} 9.8$. The breaks in the transient absorption spectra cover the scattering of the pump beam reaching the detectors. 
Table 6. Photophysical properties of 6-mercaptopurine (6MP) in PBS pH 5.5, 7.4 and borate buffer $\mathrm{pH}$ 9.8

\begin{tabular}{|c|c|c|c|}
\hline & 6MP (pH 5.5) & 6MP (pH 7.4) & 6MP (pH 9.8) \\
\hline$\lambda_{\max }(\mathbf{n m})$ & 323 & 318 & 313 \\
\hline$\varepsilon_{\max }\left(10^{3} \mathrm{M}^{-1} \mathrm{~cm}^{-1}\right)$ & $22.7 \pm 0.3$ & $19.7 \pm 0.5$ & $12.6 \pm 0.3$ \\
\hline$\tau_{1}(\mathbf{f s})$ & $180 \pm 30$ & $180 \pm 35$ & $200 \pm 35$ \\
\hline$\tau_{2}(\mathrm{ps})$ & $7 \pm 1$ & $8 \pm 1$ & $15 \pm 1$ \\
\hline$\tau_{3}(\mathrm{~ns})$ & $\gg 3 \mathrm{~ns}$ & $>>3 \mathrm{~ns}$ & $\gg 3 \mathrm{~ns}$ \\
\hline$\varepsilon_{\mathrm{T}}\left(\mathrm{M}^{-1} \mathbf{c m}^{-1}\right)$ & $\begin{array}{c}5362 \\
(492 \mathrm{~nm})\end{array}$ & $\begin{array}{c}5451 \\
(475 \mathrm{~nm})\end{array}$ & nd \\
\hline$\phi_{\mathrm{T}}$ & $1.0 \pm 0.2$ & $1.0 \pm 0.2$ & nd \\
\hline$\tau_{\mathrm{T}}(\mu \mathrm{s})$ & $\begin{array}{c}3.5 \pm 0.2 \\
(0.2 \mathrm{mM})\end{array}$ & $\begin{array}{c}0.95 \pm 0.07 \\
(0.5 \mathrm{mM})\end{array}$ & nd \\
\hline$\phi_{\Delta}(\text { air })^{\mathrm{a}}$ & $0.19 \pm 0.02^{\mathrm{c}}$ & $0.11 \pm 0.01^{\mathrm{d}}$ & nd \\
\hline$\phi_{\Delta}\left(\mathbf{O}_{2}\right)^{\mathrm{a}}$ & $0.29 \pm 0.02^{\mathrm{c}}$ & $0.16 \pm 0.02^{\mathrm{d}}$ & nd \\
\hline
\end{tabular}

$\mathrm{nd}=$ not determined; $\lambda_{\max }=$ steady-state absorption maximum; $\varepsilon_{\max }=$ steady-state absorption coefficient at absorption maximum; $\lambda_{\mathrm{T}}=$ transient absorption maxima; $\varepsilon_{\mathrm{T}}=$ triplet-triplet molar absorption coefficient; $\phi_{\mathrm{T}}=$ triplet quantum yield; $\tau_{\mathrm{T}}=$ triplet decay lifetime at the reported thiobase concentration under $\mathrm{N}_{2}$-saturated conditions; $\phi_{\Delta}$ (air) = singlet oxygen yield under air-saturated conditions; $\phi_{\Delta}\left(\mathrm{O}_{2}\right)=$ singlet oxygen yield under $\mathrm{O}_{2}$-saturated conditions; ${ }^{\text {a }}$ measured in TRIS buffer, $\mathrm{D}_{2} \mathrm{O}$ at $\mathrm{pH} 7.5$; ${ }^{\mathrm{b}}$ estimated triplet-decay lifetime from the global fit analysis; ${ }^{\mathrm{c}}$ measured in TRIS buffer, $\mathrm{D}_{2} \mathrm{O}$ at $\mathrm{pH} 5.5 ;{ }^{\mathrm{d}}$ measured in TRIS buffer, $\mathrm{D}_{2} \mathrm{O}$ at $\mathrm{pH}$ 7.5;

\subsubsection{Aza}

Figure 4 presents the transient absorption spectra for Aza exciting at $334 \mathrm{~nm}$ in PBS at $\mathrm{pH} 5.5$ and 7.4, and in borate buffer at $\mathrm{pH}$ 9.8. Figure 5 presents the transient absorption spectra for Aza exciting at $316 \mathrm{~nm}$ in PBS pH 7.4 and in acetonitrile, respectively. Within the initial 500 fs, excitation of Aza at 334 or $316 \mathrm{~nm}$ leads to transient spectra with maxima at ca. 350 and

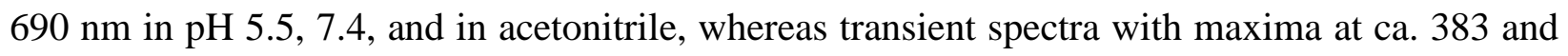
$695 \mathrm{~nm}$ are observed in $\mathrm{pH}$ 9.8. The transient species decays to populate a long-lived transient species that last longer than 3 ns. As shown in Figure S9, the timescale leading to the population of this long-lived transient species varies with $\mathrm{pH}$ and in going from PBS to acetonitrile. The lifetimes extracted from the global and target analyses are reported in Table 7 and the EADS are reported in Figures S10 and S11, respectively. 

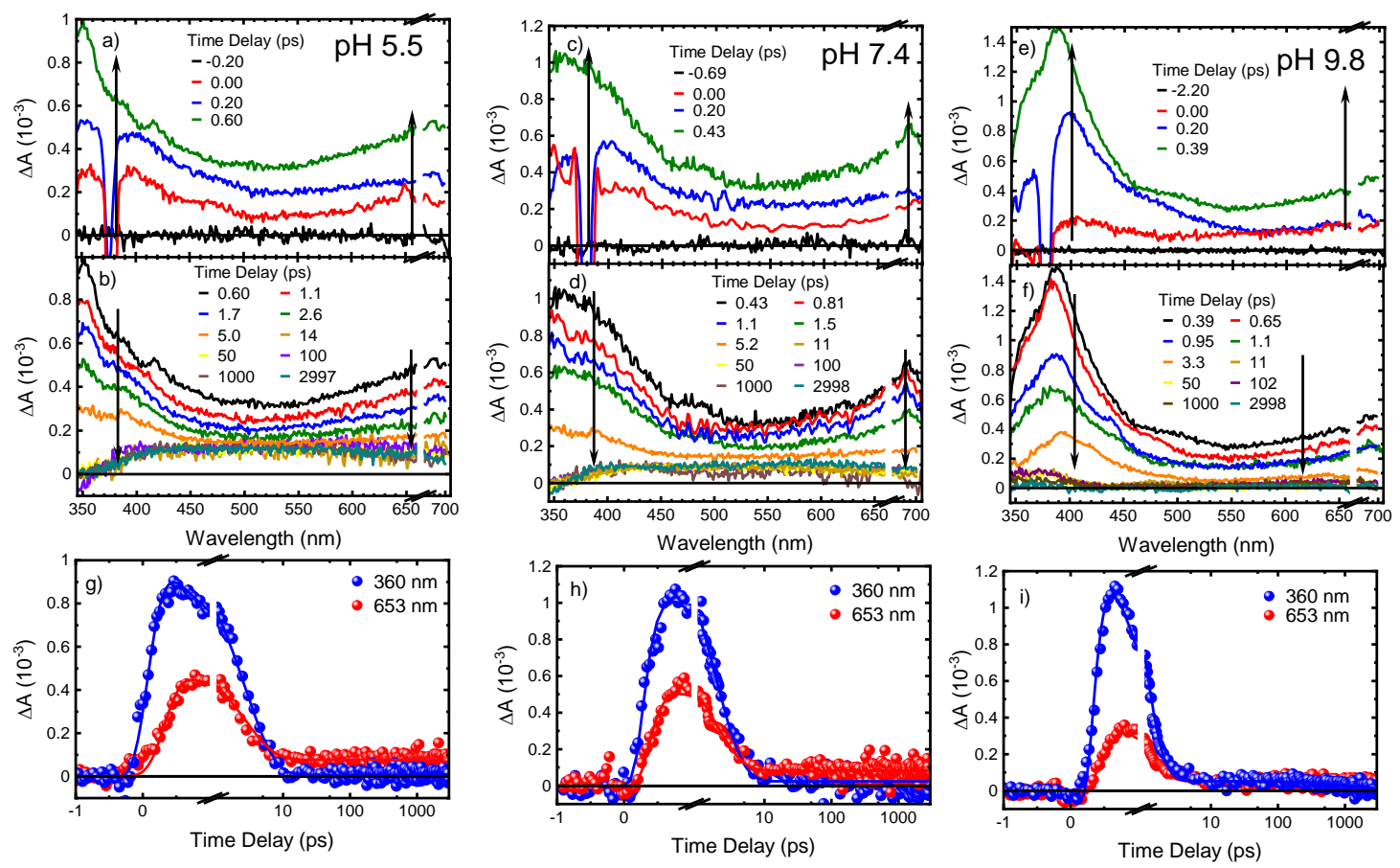

Figure 4. Transient absorption spectra of Aza $\left(\lambda_{\mathrm{exc}}=334 \mathrm{~nm}\right)$ in PBS at $\mathrm{pH} 5.5(\mathrm{a}, \mathrm{b})$ and $\mathrm{pH} 7.4(\mathrm{c}, \mathrm{d})$, and in borate buffer at $\mathrm{pH} 9.8$ (e,f). Representative decay traces in $\mathrm{g}$ ) at $\mathrm{pH} 5.5, \mathrm{~h}$ ) at $\mathrm{pH} 7.4$ and i) at $\mathrm{pH}$ 9.8. The breaks in transient absorption spectra conceal the scattering of the pump reaching the detectors. Note that the stimulated Raman emission from the water solvent is observed within the cross correlation of the pump and probe beams in panels (a, c, and e). 

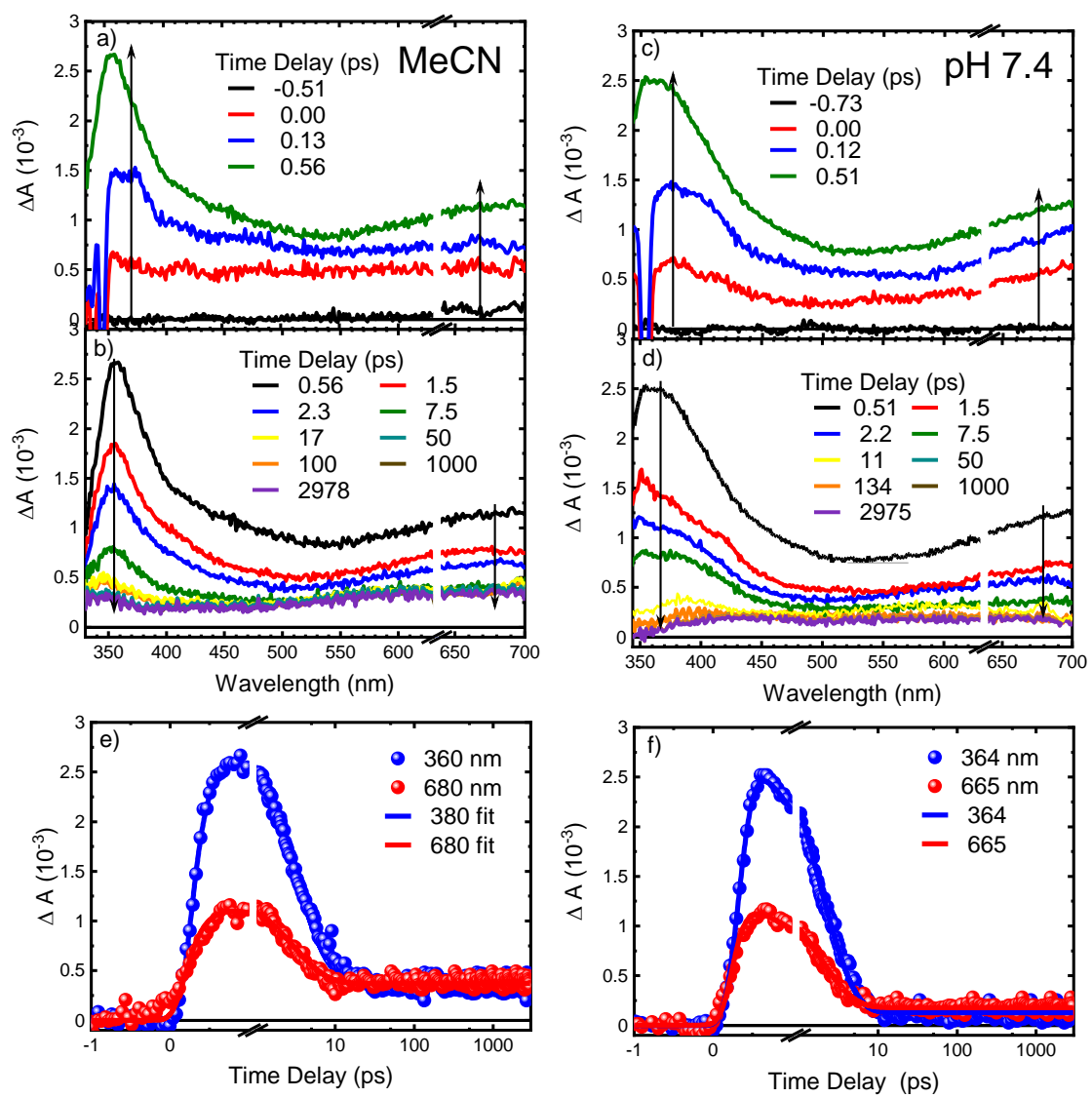

Figure 5. Transient absorption spectra of Aza in acetonitrile (MeCN; a, b) and pH 7.4 (c,d) following excitation at $316 \mathrm{~nm}$. Representative decay traces in e) acetonitrile and f) at $\mathrm{pH}$ 7.4. The breaks in the transient absorption spectra conceal the scattering of the pump reaching the detectors. Note that the stimulated Raman emission from the solvent is observed within the cross correlation of the pump and probe beams in panels (a and c).

Table 7. Photophysical properties of Aza at pH 5.5 and 7.4 in PBS, borate buffer at pH 9.8, and in acetonitrile

\begin{tabular}{ccccc}
\hline & Aza $(\mathbf{p H} 5.5)$ & Aza $(\mathbf{p H} 7.4)$ & Aza $(\mathbf{p H ~ 9 . 8 )}$ & Aza (acetonitrile) \\
\hline $\boldsymbol{\lambda}_{\max }(\mathbf{n m})$ & 279 & 281 & 285 & 280 \\
$\boldsymbol{\varepsilon}_{\mathbf{m a x}}\left(\mathbf{1 0}^{\mathbf{3}} \mathbf{M}^{-1} \mathbf{c m}^{-1}\right)$ & $14.8 \pm 0.4$ & $14.4 \pm 0.2$ & $11.7 \pm 0.3$ & $12.0 \pm 0.3$ \\
$\boldsymbol{\tau}_{\mathbf{1}}(\mathbf{p s})$ & $2.7 \pm 0.3^{\mathrm{a}}$ & $1.9 \pm 0.4^{\mathrm{a}}$ & $1.5 \pm 0.4^{\mathrm{a}}$ & $3.4 \pm 0.3^{\mathrm{b}}$ \\
$\boldsymbol{\tau}_{\mathbf{2}}(\mathbf{n s})$ & $>>3 \mathrm{~ns}^{\mathrm{a}}$ & $>>3 \mathrm{~ns}^{\mathrm{a}}$ & $>>3 \mathrm{~ns}^{\mathrm{a}}$ & $>>3 \mathrm{~ns}^{\mathrm{b}}$ \\
$\boldsymbol{\lambda}_{\mathbf{T}}(\mathbf{n m})$ & $353^{\mathrm{a}}$ & $358^{\mathrm{a}}$ & $384^{\mathrm{a}}$ & $360^{\mathrm{b}}$ \\
$\phi_{\Delta}(\mathbf{a i r})$ & $694^{\mathrm{a}}$ & $694^{\mathrm{a}}$ & $\sim 694^{\mathrm{a}}$ & $680^{\mathrm{b}}$ \\
& $\mathrm{nd}$ & $<0.01^{\mathrm{c}}$ & $\mathrm{nd}$ & $0.002 \pm 0.001$
\end{tabular}

$\mathrm{nd}=$ not determined; $\lambda_{\max }=$ steady-state absorption maximum; $\varepsilon_{\max }=$ steady-state absorption coefficient at absorption maximum; $\lambda_{\mathrm{T}}=$ transient absorption maxima. ${ }^{\mathrm{a}}$ measured upon excitation with $334 \mathrm{~nm} .{ }^{\mathrm{b}}$ measured upon excitation with $316 \mathrm{~nm} .{ }^{c}$ measured in TRIS buffer, $\mathrm{D}_{2} \mathrm{O}$ at $\mathrm{pH} 7.5$. 


\subsection{Singlet Oxygen Yields for 6MP and Aza}

Time-resolved near-infrared emission spectroscopy was used to quantify the singlet oxygen yields. Figure 6 shows representative ${ }^{1} \mathrm{O}_{2}$ emission decay traces for $6 \mathrm{MP}$, Aza, and the phenalenone standard, while Tables 6 and 7 collect the determined singlet oxygen quantum yields. Singlet oxygen quantum yields of 0.19 and 0.11 were determined for $6 \mathrm{MP}$ at $\mathrm{pH} 5.5$ and 7.5 under air-saturated conditions, respectively. Under oxygen-saturated conditions, the singlet oxygen quantum yields increase to 0.29 and 0.16 at $\mathrm{pH} 5.5$ and 7.5 , respectively. In the case of Aza, singlet oxygen quantum yields of $<0.01$ and 0.001 were determined at $\mathrm{pH} 7.5$ and in acetonitrile, respectively. The 10-fold lower yield of Aza compared to 6MP evidence that the covalent attachment of a nitroimidazole group significantly affects its photochemistry.
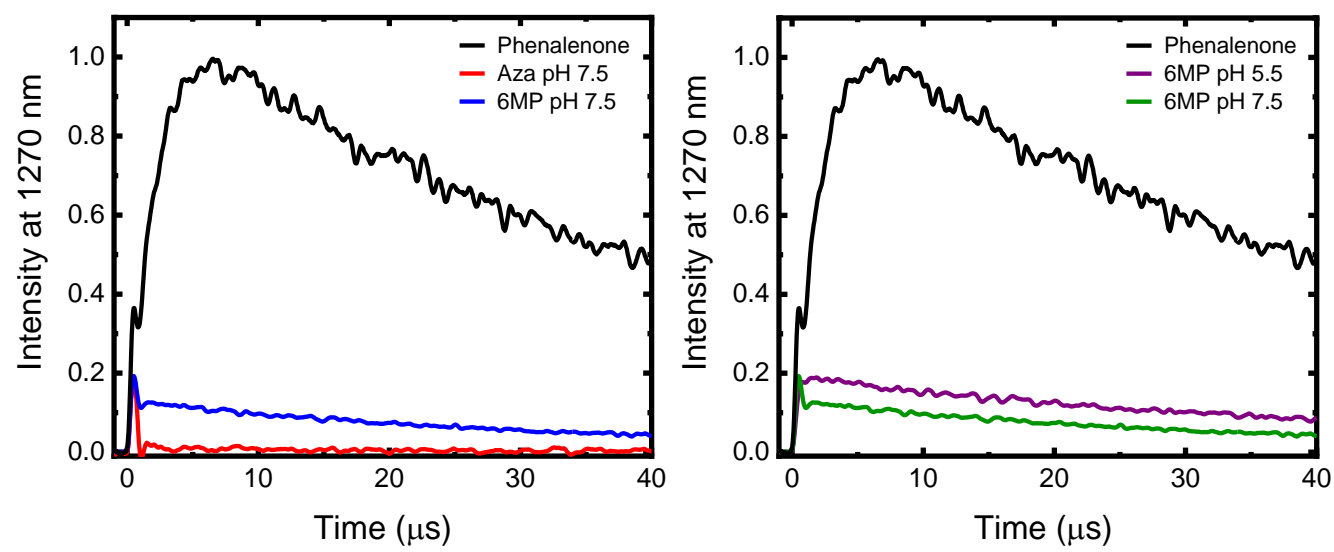

Figure 6. Time-resolved ${ }^{1} \mathrm{O}_{2}$ emission traces for 6-mercaptopurine (6MP), azathioprine (Aza), and phenalenone (standard) in air saturated TRIS buffer, $\mathrm{D}_{2} \mathrm{O}$ at $\mathrm{pH} 5.5$ and 7.5.

\subsection{Nanosecond Transient Absorption of 6MP}

Nanosecond transient absorption experiments were performed at $\mathrm{pH} 5.5$ and 7.4 to determine the triplet decay lifetimes of both the neutral and the deprotonated forms of 6MP. Figure 7 shows representative triplet decay traces. Only one decay lifetime was needed to fit the data at $\mathrm{pH} 5.5(3.5 \pm 0.2 \mu \mathrm{s})$, whereas two decay lifetimes were required at $\mathrm{pH} 7.4(0.95 \pm 0.07$ 
$\mu$ s and $3.5 \mu \mathrm{s}$ ), the second lifetime was held to the value obtained for the neutral form at $\mathrm{pH}$ 5.5. The relative fractions of the deprotonated and the neutral forms extracted from the amplitudes of the bi-exponential fit are $68 \%$ and $32 \%$, respectively, which are in excellent agreement with those predicted using the Henderson-Hasselbalch relationship (67\% and 33\%).

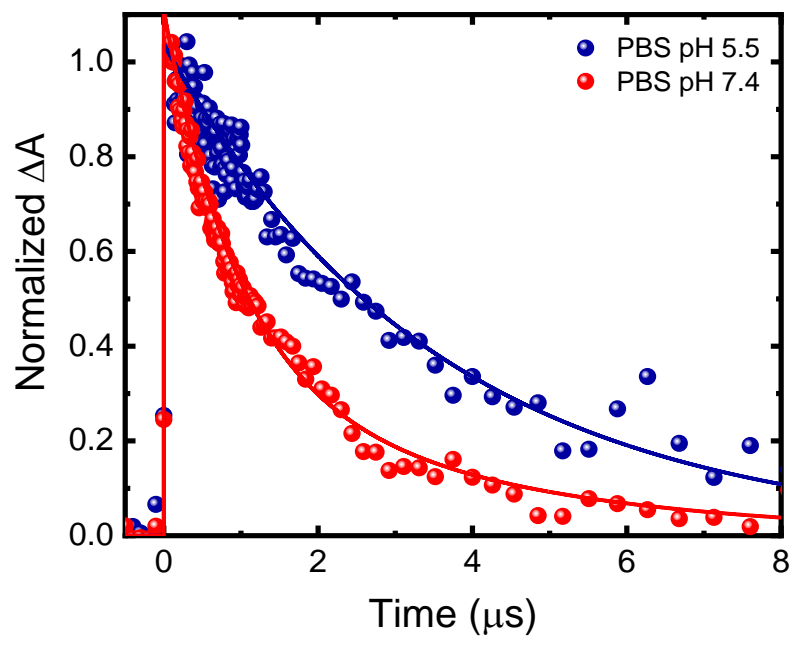

Figure 7. Triplet decay traces for 6MP following excitation at $334 \mathrm{~nm}$ in $\mathrm{PBS}$ at $\mathrm{pH} 5.5$ and 7.4. A single exponential decay function was required to fit the decay trace at $\mathrm{pH}$ 5.5, whereas a two-exponential decay function was required to fit the decay trace at $\mathrm{pH}$ 7.4. See main text for details.

\section{DISCUSSION}

Aza and 6MP figure in the World Health Organization top one hundred list of essential medicines and are prescribed for the treatment of leukemia, autoimmune conditions, and as immunosuppressants. ${ }^{1}$ However, research has also shown that UVA irradiation of cells treated with Aza and 6MP causes oxidatively generated damage to both the genome and the proteome. ${ }^{7}$

${ }^{8}$ Half of the observed oxidatively generated DNA damage in cells treated with Aza or 6thioguanine (6tGua) has been demonstrated to originate from the direct photoactivation of the metabolized 6tGuo nucleotide in the intracellular pool. ${ }^{9}$ Similarly, excitation of 6tGua with UVA radiation has been shown to induce oxidatively generated damage to nucleotides in the 
intracellular pool, ${ }^{9}$ forming the 2 '-deoxy-8-oxoguanosine nucleotide, which can then be incorporated into cellular DNA, ${ }^{19}$ leading to indirect DNA damage. Therefore, it is important to examine whether Aza and 6MP themselves can induce oxidatively generated damage to cellular DNA in the intracellular pool and to the proteome. To narrow this gap in knowledge, the excited state dynamics and the photochemical properties of the Aza and 6MP prodrugs were investigated in this study to elucidate their electronic relaxation mechanisms, as well as the plausible formation of reactive triplet states and reactive oxygen species in aqueous solutions.

\subsection{Electronic Relaxation Mechanism in 6MP}

We first focus on elucidating the electronic relaxation mechanism for 6MP in aqueous solution at three different pHs. The evolution-associated difference spectra extracted from a global and target analysis of the transient absorption data reported in Figure 3 satisfactorily captures all the observed spectral changes (see Figure S8). Hence, a direct comparison of the evolution-associated difference spectra with the calculated excited-state absorption spectra for the $S_{2}\left(\pi \pi^{*}\right), S_{1}\left(n \pi^{*}\right), T_{2}\left(n \pi^{*}\right)$ and $T_{1}\left(\pi \pi^{*}\right)$ states, reported in Figure $S 5$, is expected to provide crucial information about the excited states that participate in the relaxation mechanism.

Figure S12 compares the extracted evolution-associated difference spectra for the first two components with the calculated excited-state absorption spectra of the $S_{1}\left(n \pi^{*}\right)$ state and with a linear combination of the absorption spectra for the two $T_{1}\left(\pi \pi^{*}\right)$ minima obtained from the calculations. Based on these results, the transient signal that appears around time zero in Figure 3 can be confidently assigned to excited state absorption from the $S_{1}\left(n \pi^{*}\right)$ state, while the subsequent longer-lived transient species can be satisfactorily assigned to a linear combination of the absorption spectra of both $\mathrm{T}_{1}\left(\pi \pi^{*}\right)$ minima. Furthermore, the spectrum of this long-lived transient species closely resembles the one reported for $6 \mathrm{MP}$ on the microsecond time scale, ${ }^{46}$ 
which has been assigned to the absorption spectrum of the $T_{1}$ state. Hence, using the singlet depletion and relative actinometry methods, the triplet yield of 6MP was determined in this study to be $1.0 \pm 0.2$ at both $\mathrm{pH} 5.5$ and 7.4 in PBS, relative to 6-thioguanosine. The triplet yield was not obtained for $6 \mathrm{MP}$ in $\mathrm{pH} 9.8$ because no clear bleaching signal can be observed due to the blue shifted absorption spectrum (Figure 1a) of $6 \mathrm{MP}$ at $\mathrm{pH}$ 9.8. However, based on the similarities of the transient spectra regardless of the $\mathrm{pH}$ used (Figure 3), a near-unity triplet yield is also expected for $6 \mathrm{MP}$ at $\mathrm{pH} 9.8$. The triplet yield reported in this study is in good agreement with a triplet yield of 0.99 that was measured previously for 6MP in tetrahydrofuran using an energy transfer method. ${ }^{57}$ The efficient population of the triplet state is also consistent with the room-temperature phosphorescence reported in Figures S1 to S3 for 6MP. Hence, the transient spectra reported in Figure 3 evidence that the $\mathrm{T}_{1}$ state is populated on an ultrafast time scale (i.e., within $\tau_{1}$ ), which is further supported by the vertical excitation energies and the SOCs reported in Tables 1 and 2.

The calculations further predict that the triplet manifold of 6MP is populated through two competitive intersystem crossing pathways. The first pathway is intersystem crossing directly from the $S_{2}\left(\pi \pi^{*}\right)$ state to the $T_{2}\left(n \pi^{*}\right)$ state, followed by internal conversion to the $T_{1}\left(\pi \pi^{*}\right)$ state. The second pathway involves internal conversion from the $S_{2}\left(\pi \pi^{*}\right)$ state to the $S_{1}\left(n \pi^{*}\right)$ state, followed by intersystem crossing to the $T_{1}\left(\pi \pi^{*}\right)$ state. The participation of the first pathway can be supported by the slight blueshift and increase in intensity of the transient species as a function of time delay (Figure 3). This slight blueshift and increase in intensity is characteristic of conformational relaxation and vibrational cooling dynamics. The excited state optimizations predict that the sulfur atom in the $T_{2}\left(n \pi^{*}\right)$ state exhibits significant puckering compare to that of the $T_{1}\left(\pi \pi^{*}\right)$ planar minima (Figure S8). Thus, it is possible that the slight blueshift and increase 
in intensity observed in the transient spectra is associated not only with vibrational cooling in the $\mathrm{T}_{1}\left(\pi \pi^{*}\right)$ state $\left(\Delta \mathrm{E}\left(\mathrm{T}_{2}-\mathrm{T}_{1}\right) \sim 1 \mathrm{eV}\right)$, but also with conformational relaxation dynamics upon internal conversion from the $T_{2}\left(n \pi^{*}\right)$ state to the $T_{1}\left(\pi \pi^{*}\right)$ planar minima. We further note that large blueshift and increase in intensity are not observed in the transient spectra. This could be due to a significant fraction of the $\mathrm{T}_{2}\left(\mathrm{n} \pi^{*}\right)$ state population being able to internally convert to the out-of-plane $\mathrm{T}_{1}\left(\pi \pi^{*}\right)$ minima, in which the sulfur atom is also out of the plane of the purine chromophore and conformational relaxation is not expected to play a major role. The participation of the indirect intersystem crossing pathway is supported by the observation that the calculated absorption spectrum of the $S_{1}\left(n \pi^{*}\right)$ state is in good agreement with the experimental transient absorption species initially observed in Figure 3. Hence, we conclude that both direct and indirect intersystem crossing pathways contribute to the efficient population of the $\mathrm{T}_{1}\left(\pi \pi^{*}\right)$ state of $6 \mathrm{MP}$.

The triplet state of 6MP decays with a single lifetime of $3.5 \pm 0.2 \mu \mathrm{s}$ at $\mathrm{pH} 5.5$ (Figure 7), whereas it decays with two lifetimes of $3.5 \pm 0.2 \mu$ s and $0.95 \pm 0.07 \mu$ s at $\mathrm{pH}$ 7.4. This supports the idea that the $3.5 \pm 0.2 \mu$ s lifetime corresponds to the triplet decay lifetime of the neutral form, whereas the $0.95 \pm 0.07 \mu$ s lifetime corresponds to the triplet decay of the deprotonated form of 6MP. In Figure 6, the triplet state was demonstrated to sensitize the generation of ${ }^{1} \mathrm{O}_{2}$ with an efficiency of $19 \%$ and $11 \%$ at $\mathrm{pH} 5.5$ and 7.5 , respectively. These values are low considering the near-unity triplet yield estimated for both the neutral and deprotonated forms of 6MP in this study. However, as described in the results section, and as has been previously proposed for 6tGua and 6tGuo, ${ }^{16,17}$ molecular oxygen can quench the triplet state of $6 \mathrm{MP}$ by forming reactive oxygen species other than singlet oxygen ${ }^{46}$ Hence, to shed light on this possibility, the Gibbs free energies associated with the electron transfer process $\left(\Delta \mathrm{G}_{\mathrm{ET}}\right)$ from the ${ }^{3} \pi \pi^{*}$ state of both the 
neutral and deprotonated forms of $6 \mathrm{MP}$ and $\mathrm{O}_{2}$ were calculated for both the planar and out-ofplane $\mathrm{T}_{1}$ minima (Table 5). According to Schweitzer et al., a charge transfer complex can dominate the interaction between $\mathrm{a}^{3} \pi \pi^{*}$ state and $\mathrm{O}_{2}$ when the $\Delta \mathrm{G}_{\mathrm{ET}}$ value is more negative than $-30 \mathrm{~kJ} / \mathrm{mol}^{36}$ As shown in Table 5, $\Delta \mathrm{G}_{\mathrm{ET}}$ of $-129(-81.2)$ and $-374(-365.7) \mathrm{kJ} / \mathrm{mol}$ were estimated for the out-of-plane (planar) minima of the neutral and the deprotonated forms of 6MP, respectively. ${ }^{8,} 30$ These Gibbs free energy estimates support the idea that a significant fraction of the triplet state could be quenched by electron transfer to generate superoxide radicals, with the out-of-plane $\mathrm{T}_{1}$ minima exhibiting higher probability for electron transfer to $\mathrm{O}_{2}$ than the planar minima.

Recently, Bai and Barbatti ${ }^{53}$ proposed that the topology of the $\mathrm{T}_{1}\left(\pi \pi^{*}\right)$ potential energy surface of the thiobases is characterized by two minima, in agreement with our computational results and those by others. ${ }^{53-55}$ It was further proposed that depending on the energetic balance between the two minima, the rate determining step can be a slow transition between both minima that favors the sensitization of singlet oxygen when the out-of-plane minimum is higher in energy than the other minimum, which they labeled as ring distorted minimum, or faster intersystem crossing back to the ground-state when the distorted minimum is higher in energy than the out-of-plane minimum. According to our calculations, the energy gap between the outof-plane and planar minima is $\sim 0.5 \mathrm{eV}$ for the neutral $6 \mathrm{MP}$, whereas the energy gap between both minima is $\sim 0.08 \mathrm{eV}$ for the deprotonated form of $6 \mathrm{MP}$. In both cases, the planar minimum is lower in energy than the out-of-plane minimum, which should favor reaction with $\mathrm{O}_{2}$ over intersystem crossing to the ground state. Then, the generation of the superoxide radical can partially explain the relatively low yields of ${ }^{1} \mathrm{O}_{2}$ generation observed in this study. We also note that the larger the energy gap between both minima, the longer the triplet decay lifetime and the 
higher the singlet oxygen quantum yield are. Hence, the energy gap between both $\mathrm{T}_{1}\left(\pi \pi^{*}\right)$ minima in the neutral and the deprotonated forms of 6MP, together with the favorable probability to form superoxide radicals (Table 5), may explain the relatively low yields of ${ }^{1} \mathrm{O}_{2}$ generation and the reduction in ${ }^{1} \mathrm{O}_{2}$ quantum yield in going from the neutral form of $6 \mathrm{MP}$ to the deprotonated form.

Even though the magnitude of the triplet decay lifetime of the neutral and the deprotonated forms of $6 \mathrm{MP}$ is significantly different, the pathways leading to the reactive triplet state population are expected to be the same. This is supported by the observation that the spectral features in the transient data, the VEEs, and the SOCs are nearly the same regardless of whether the neutral or the deprotonated form is investigated. The 3.7-fold longer triplet decay lifetime observed for the neutral relative to the deprotonated species is proposed to be due to the larger energy gap between the triplet minima to access the intersystem crossing region leading to the repopulation of the ground state. The singlet oxygen yield is larger at $\mathrm{pH} 5.5$ than at $\mathrm{pH} 7.4$ showing that the neutral species generates singlet oxygen more efficiently than the deprotonated species. This is consistent with the longer triplet decay lifetime of the neutral species compared to the triplet decay lifetime of the deprotonated species. It also seems to be consistent with the larger driving force for electron transfer from the triplet state of the deprotonated species to molecular oxygen compared to the triplet state of the neutral species, as suggested by the calculations reported in Table 5 .

Finally, we note that the DFT calculations reported in this study predict a notoriously low VIE of $3.4 \mathrm{eV}$ for the deprotonated form of $6 \mathrm{MP}$, which leads to the prediction of a negative oxidation potential energy (Table 5). We think that this is an artifact of DFT that results in the underestimation of the VIE for the deprotonated form at this level of theory. DFT is known to 
underestimate the energies of anionic species, ${ }^{58,59}$ hence, it would not be surprising that it may also underestimate the VIE of the deprotonated form of 6MP. Indeed, similar VIE calculations performed using other functionals (PBE0, M06, and B3PW91) lead to similarly low values $(\sim 3.5$ $\mathrm{eV}$, Table S1), but the calculation of the VIE using the MP2 level of theory leads to a ca. $1 \mathrm{eV}$ higher ionization energy for the deprotonated form of $6 \mathrm{MP}(4.4 \mathrm{eV})$. This result supports our interpretation of the VIE using DFT and caution against overinterpretation of the results presented in Table 5 for the deprotonated form of $6 \mathrm{MP}$.

\subsection{Electronic Relaxation Mechanism in Aza}

Excitation of Aza at 334 or $316 \mathrm{~nm}$ leads to the observation of transient absorption spectra with maxima at ca. 350 and $690 \mathrm{~nm}$ in $\mathrm{pH} 5.5,7.4$, and in acetonitrile within the initial ca. $0.5 \mathrm{ps}$, while it leads to the observation of transient spectra with maxima at ca. 383 and 695 $\mathrm{nm}$ in $\mathrm{pH}$ 9.8. The transient spectra decay to populate a minor, long-lived transient species that last longer than 3 ns. Considering the VEEs reported in Table 3 for Aza, the transient absorption species observed within $0.5 \mathrm{ps}$ is assigned to the excited state absorption of the $\mathrm{S}_{1}\left(\pi \pi^{*}, \mathrm{CT}\right)$ state for both the neutral and the deprotonated forms of Aza. It should be remarked that both $S_{1}\left(\pi \pi^{*}\right.$, CT) and $S_{2}\left(\pi \pi^{*}, C T\right)$ states are expected to be simultaneously populated upon excitation of Aza at 334 or $316 \mathrm{~nm}$ (see Figure $\mathrm{S} 7$ for details) independent of $\mathrm{pH}$ (e.g., $~ 95 / 77 \%$ directly populates the $S_{1}$ and $\sim 5 / 23 \%$ the $S_{2}$ state upon excitation of Aza with 334/316 nm at $\mathrm{pH} 5.5$, while $\sim 79 / 34 \%$ directly populates the $S_{1}$ and $\sim 21 / 66 \%$ populates the $S_{2}$ state upon excitation with $334 / 316 \mathrm{~nm}$ at $\mathrm{pH}$ 9.8). Irrespective of the exact fraction of the population that reaches both excited singlet states, the population reaching the $S_{2}$ state is expected to internally convert in an ultrafast time scale to the $S_{1}\left(\pi \pi^{*}, C T\right)$ state (i.e., faster than the IRF of the spectrometer of ca. 
$250 \mathrm{fs}$ ). As shown in Figure 2, the $\mathrm{S}_{1}$ state has significant (>42\%) CT character in both the neutral and the deprotonated forms. Thus, it is proposed that most of its population leads to ultrafast intramolecular charge separation, followed by charge recombination repopulating the ground state within $\tau_{1}$. Indeed, rapid intramolecular charge recombination is often observed in molecules having intramolecular donor and acceptor moieties such as $\mathrm{Aza}^{35}$ where the interchromophoric distance is small and the charges cannot separate for long periods across the donor-acceptor pair to stabilize the charge separation state. ${ }^{35,60,61}$

The small-amplitude residual signals observed after the primary charge recombination event is completed and that decays in longer than $3 \mathrm{~ns}$ independent of the $\mathrm{pH}$ of the solution in range from 5.5 to 9.8 (Figure 4), is assigned to the $\mathrm{T}_{1}$ state of both the neutral (at $\mathrm{pH} 5.5$ ) and the deprotonated (at $\mathrm{pH}$ 9.8) species. Relative yields of $15 \%, 7 \%$ and $3 \%$ are estimated from the transient absorption data for Aza at pH 5.5, 7.4 and 9.8, respectively (see Methods). The putative assignment of this transient species to photoproduct formation can be safely discarded because Aza did not degrade during the transient absorption experiments. The calculations also predict that singlet to triplet intersystem crossing events can effectively occur between the $S_{1} \rightarrow T_{2}, S_{2}$ $\rightarrow T_{2}, S_{2} \rightarrow T_{1}$, and $S_{2} \rightarrow T_{4}$ states for the neutral form or between the $S_{1} \rightarrow T_{2}$ and $S_{2} \rightarrow T_{2}$ for the deprotonated form of Aza (Tables 3 and 4). However, because intersystem crossing in the neutral and the deprotonated forms must compete with ultrafast charge separation and recombination in the $S_{1}\left(\pi \pi^{*}, C T\right)$ state, the triplet yields are expected to be very small, as observed. The low triplet yield is supported by the low ${ }^{1} \mathrm{O}_{2}$ yield reported in Table 7 , and by the observation of phosphorescence at $77 \mathrm{~K}^{62}$ for Aza but not at room temperature. The possibility that the triplet state of Aza can be populated is also indirectly supported by the observation of 
triplet state population in $S^{6}$-methylthioinosine, which also features a C6-S single bond, in both aqueous and acetonitrile solutions. ${ }^{63}$

Furthermore, transient absorption experiments performed for Aza in acetonitrile and in PBS at pH 7.4 in back-to-back conditions demonstrated nearly identical spectral features but a $44 \%$ slowdown of the charge recombination lifetime and a 2.6-fold increase in the amplitude of the longer-lived species in acetonitrile compared to $\mathrm{pH} 7.4$ (Figure 5). The slowdown of the charge recombination lifetime together with the increased amplitude of the long-lived species demonstrates that the initial population in the singlet manifold bifurcates to populate an intramolecular charge-transfer state and a long-lived transient species. Solvent polarity is known to affect the intramolecular charge transfer state dynamics in such systems, with highly polar solvents having a stronger interaction with the intramolecular charge-transfer state, which can result in faster solvation and subsequent decay to lower energy levels. ${ }^{64}$ It has also been reported that certain push-pull systems with less CT character are less influenced by solvent polarity, which can result in a higher prevalence of decay channels other than charge recombination, for example, intersystem crossing to the triplet state. ${ }^{65}$ 

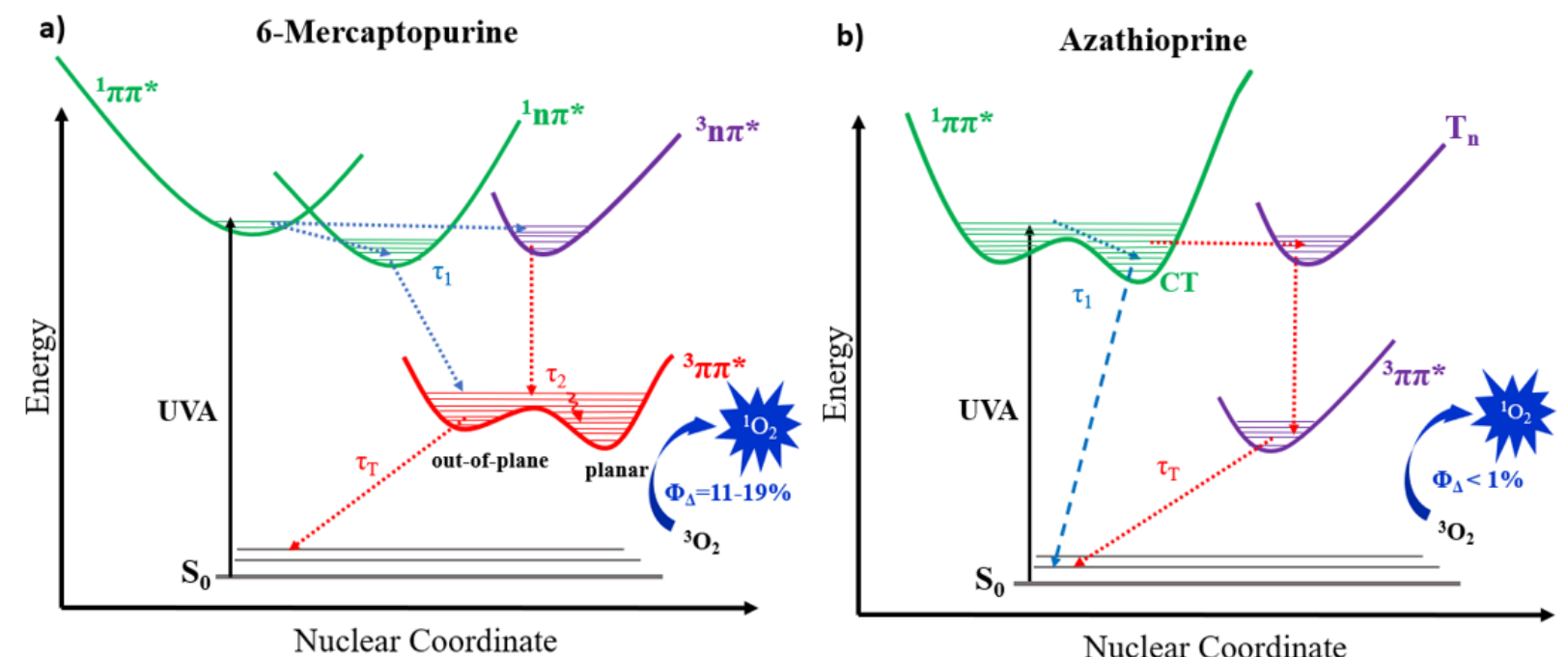

Scheme 3. Proposed electronic relaxation mechanisms for a) 6MP and b) Aza prodrugs upon UVA excitation. The planar $\mathrm{T}_{1}$ minimum is lower in energy than the out-of-plane minimum. The planar $\mathrm{T}_{1}$ is expected to facilitate the production of more ${ }^{1} \mathrm{O}_{2}$ than the out-of-plane minimum, while the out-of-plane $\mathrm{T}_{1}$ is expected to facilitate the production of more superoxide than the planar minimum. Note that the calculations predict that upon excitation at 334 or $316 \mathrm{~nm}$, and depending on the protonation status of Aza, the $S_{2}$ state can also be populated, and a small fraction of its population may also intersystem cross to the triplet manifold, while the large majority is expected to internally convert to the $S_{1}$ state (not shown in the scheme, see main text).

\section{CONCLUSIONS}

Scheme 3 summarizes the electronic relaxation mechanisms outlined in this study for the 6MP and Aza prodrugs. On the one hand, it is proposed that upon UVA photoactivation, 6MP populates the triplet manifold in ultrafast timescales $\left(\tau_{1}\right)$ by two competitive pathways. The first pathway is direct intersystem crossing from the $S_{2}\left(\pi \pi^{*}\right)$ state to the $T_{2}\left(n \pi^{*}\right)$ state. The second pathway involves internal conversion from the $S_{2}\left(\pi \pi^{*}\right)$ state to the $S_{1}\left(n \pi^{*}\right)$ state, followed by intersystem crossing to the $\mathrm{T}_{1}\left(\pi \pi^{*}\right)$ state. Both pathways agree with the El-Sayed's propensity rules. The population reaching the vibrational excited $\mathrm{T}_{1}\left(\pi \pi^{*}\right)$ state, bifurcates and populate two $\mathrm{T}_{1}\left(\pi \pi^{*}\right)$ minima within $\tau_{2}$, through internal conversion undertaking conformational relaxation and vibrational cooling dynamics in the $\mathrm{T}_{1}\left(\pi \pi^{*}\right)$ potential energy surface. After the $\mathrm{T}_{1}\left(\pi \pi^{*}\right)$ state reaches the out-of-plane and planar minima, it decays back to the ground-state with lifetimes of 
$3.5 \pm 0.2 \mu$ s and $0.95 \pm 0.07 \mu$ s for the neutral and the deprotonated species, respectively. The relatively long triplet decay lifetime in $6 \mathrm{MP}$ allows the diffusion-controlled sensitization of ${ }^{1} \mathrm{O}_{2}$ with a yield of $11 \%$ (deprotonated) to $19 \%$ (neutral) and the generation the superoxide radical.

On the other hand, excitation of Aza at $334 \mathrm{~nm}$ or $316 \mathrm{~nm}$ is proposed to result in the population of both the $S_{2}$ and the $S_{1}$ states, which the former decaying by ultrafast internal conversion to the $S_{1}$ state (not shown in Scheme $3 b$ ). The $S_{1}$ state primarily relaxes by intramolecular charge separation and charge recombination to the ground state within $\tau_{1}$, while a minor fraction of the excited state population in the singlet manifold intersystem crosses to the triplet manifold leading to the population of the $\mathrm{T}_{1}$ state with a very small yield (from ca. 15 to $3 \%$ depending on $\mathrm{pH}$ ). Even though there are quantitative differences in the rates governing the deactivation pathways of Aza depending on whether the neutral or the deprotonated form of Aza is investigated (or whether PBS or acetonitrile is used), the qualitative deactivation mechanism seems to be similar.

The central finding of this investigation is that Aza prodrug exhibits a remarkably different electronic relaxation mechanism and photochemistry than 6MP, as well as the other thiobases investigated to date. ${ }^{6,21,66,67}$ Excitation of 6MP with UVA radiation results in nearunity triplet yield, up to $30 \%{ }^{1} \mathrm{O}_{2}$ yield in saturated $\mathrm{O}_{2}$ conditions, and the plausible formation of superoxide radicals. The experimental and computational results suggest that not only the metabolites of 6MP and Aza, ${ }^{9}$ but 6MP itself can induce oxidatively generated damage within the intracellular pool. Hence, direct absorption of UVA radiation by 6MP could be another important contributing factor for the up to 250 -fold increase in basal and squamous cell carcinomas in patients. ${ }^{2}$ Conversely, excitation of Aza with UVA radiation leads to less than 3$15 \%$ triplet yield depending on the $\mathrm{pH}$ and less than $1 \%{ }^{1} \mathrm{O}_{2}$ yield. Nitroimidazole 
functionalization to form Aza destabilizes the ${ }^{1} \mathrm{n} \pi^{*}$ state in the Franck-Condon region and increases the charge transfer character of the $S_{1}\left(\pi \pi^{*}\right)$ state. The efficient intramolecular charge recombination decreases the ${ }^{1} \mathrm{O}_{2}$ yield by 10 -fold relative to $6 \mathrm{MP}$. We remark that formation of a carbon-sulfur single bond is not a sufficient condition to reduce the population of the $\mathrm{T}_{1}$ state in Aza because $S^{6}$-methylthioinosine populates the $T_{1}$ state in a high yield. ${ }^{63}$ Aza's poor photosensitization ability reveals the prospective use of interchromophoric interactions (i.e., the use of electron-withdrawing groups covalently attached to the thiocarbonyl sulfur atom) for the design of photostable prodrugs exhibiting a significant reduction in photocytotoxic side effects before drug metabolization.

\section{ACKNOWLEDGMENTS}

The authors acknowledge funding from the National Science Foundation (Grant No. CHE1800052). L.A.O-.R. also acknowledge the NSF-AGEP and the NOA-AGEP programs for support. Some of the initial results reported in this study were supported by the CAREER program of the National Science Foundation (Grants No. CHE-1255084 and CHE-1539808). This work made use of the High Performance Computing Resource in the Core Facility for Advanced Research Computing at Case Western Reserve University.

\section{Authors' Contributions (Based on CRediT Recommendations)}

Luis A. Ortiz-Rodríguez: investigation, formal analysis, writing-original draft, writing review and editing

Glesmarie Ortiz-Zayas: investigation, formal analysis, writing-original draft, writing review and editing

Marvin Pollum: investigation, formal analysis, writing-original draft, writing review and editing Sean J. Hoehn: investigation, writing review and editing 
Steffen Jockusch: investigation, formal analysis, writing review and editing

Carlos E. Crespo-Hernández: conceptualization, funding acquisition, project administration, resources, supervision, visualization, validation, writing-review and editing

\section{REFERENCES}

1. The selection and use of essential medicines; 1006; World Health Organization: Geneva, Switzerland, 2017.

2. $\quad$ Euvrard, S.; Kanitakis, J.; Claudy, A., Skin cancers after organ transplantation. N. Engl. J. Med. 2003, 348, (17), 1681-1691.

3. O'Donovan, P.; Perrett, C. M.; Zhang, X.; Montaner, B.; Xu, Y.-Z.; Harwood, C. A.; McGregor, J. M.; Walker, S. L.; Hanaoka, F.; Karran, P., Azathioprine and UVA light generate mutagenic oxidative DNA damage. Science 2005, 309, 1871-1874.

4. $\quad$ Perrett, C. M.; Walker, S. L.; O'Donovan, P.; Warwick, J.; Harwood, C. A.; Karran, P.; McGregor, J. M., Azathioprine treatment sensitizes human skin to ultraviolet A radiation. Br. $J$. Dermatol. 2008, 159, 198-204.

5. Attard, N. R.; Karran, P., UVA photosensitization of thiopurine and skin cancer in organ transplant recipients. Photochem. Photobiol. Sci. 2012, 11, 62-68.

6. Ortiz-Rodríguez, L. A.; Crespo-Hernández, C. E., Thionated organic compounds as emerging heavy-atom-free photodynamic therapy agents. Chem. Sic. 2020, 11, 11113-11123.

7. Gueranger, Q.; Li, F.; Peacock, M.; Larnicol-Fery, A.; Brem, R.; Macpherson, P.; Egly, J.-M.; Karran, P., Protein oxidation and DNA repair inhibition by 6-thioguanine and UVA radiation. J. Invest. Dermatol. 2014, 134, 1408-1417.

8. Brem, R.; Daehn, I.; Karran, P., Efficient DNA interstrand crosslinking by 6-thioguanine and UVA radiation. DNA Repair 2011, 10, 869-876.

9. $\quad$ Cooke, M. S.; Duarte, T. L.; Cooper, D.; Chen, J.; Nandagopal, S.; Evans, M. D., Combination of azathioprine and UVA irradiation is a major source of cellular 8-oxo-7,8dyhydro-2'-deoxyguanosine. DNA Repair 2008, 7, 1982-1989.

10. Brem, R.; Karran, P., Multiple forms of DNA damage caused by UVA photoactivation of DNA 6-thioguanine. Photochem. Photobiol. 2012, 88, 5-13.

11. Zhang, Y.; Zhu, X.; Haygood, M. T.; Gao, R., Direct observation and quantitative characterization of singlet oxygen in aqueous solution upon UVA excitation of 6-thioguanines. $J$. Phys. Chem. B 2011, 115, 1889-1894.

12. Zhang, Y.; Barnes, A. N.; Zhu, X.; Campbell, N. F.; Gao, R., Quantification of thiopurine/UVA-induced singlet oxygen production. J. Photochem. Photobiol. A 2011, 224, 1624.

13. Reichardt, C.; Guo, C.; Crespo-Hernández, C. E., Excited-state dynamics in 6thioguanosine from the femtosecond to microsecond time scale. J. Phys. Chem. B 2011, 115, 3263-3270.

14. Martínez-Fernández, L.; González, L.; Corral, I., An ab initio mechanism for efficient population of triplet states in cytotoxic sulfur substituted DNA bases: the case of 6-thioguanine. Chem. Commun. 2012, 48, 2134-2136.

15. Martínez-Fernández, L.; Corral, I.; Granucci, G.; Persico, M., Competing ultrafast intersystem crossing and internal conversion: a time resolved picture for the deactivation of 6thioguanine. Chem. Sci. 2014, 5, 1336-1347. 
16. Pollum, M.; Ortiz-Rodríguez, L. A.; Jockusch, S.; Crespo-Hernández, C. E., The triplet state of 6-thio-2-deoxyguanosine: intrinsic properties and reactivity toward molecular oxygen. Photochem. Photobiol. 2016, 92, 286-292.

17. Ashwood, B.; Jockusch, S.; Crespo-Hernández, C. E., Excited-state dynamics of the thiopurine prodrug 6-thioguanine: Can N9-glycosylation affect its phototoxic activity? Molecules 2017, 22, (3), 379.

18. Pollum, M.; Martínez-Fernández, L.; Crespo-Hernández, C. E., Photochemistry of nucleic acid bases and their thio-and aza-analogues in solution. In Photoinduced Phenomena in Nucleic Acids I, Barbatti, M. B., A. C.; U. Susanne, Ed. Springer, Cham: Switzerland, 2015; Vol. 355, pp 245-327.

19. Arecco, A.; Mun, B.; Mathews, C. K., Deoxyribonucleotide pools as targets for mutagenesis by N-methyl-N-nitrosourea. Mutat. Res. 1988, 200, 165-175.

20. Arslancan, S.; Martínez-Fernández, L.; Corral, I., Photophysics and Photochemistry of Canonical Nucleobases' Thioanalogs: From Quantum Mechanical Studies to Time Resolved Experiments. Molecules 2017, 22, 998.

21. Ashwood, B.; Pollum, M.; Crespo-Hernández, C. E., Photochemical and photodynamical properties of sulfur-substituted nucleic acid bases. Photochem. Photobiol. 2019, 95, (1), 33-58.

22. Brister, M. M.; Gustavsson, T.; Crespo-Hernández, C. E., Excited-state lifetimes of sulfur-substituted DNA and RNA monomers probed using the femtosecond fluorescence upconversion technique. Molecules 2020, 25, 584.

23. Adamo, C.; Barone, V., Toward reliable density functional methods without adjustable parameters: the PBE0 model. J. Chem. Phys. 1999, 110, 6158-6170.

24. Adamo, C.; Scuseria, G. E.; Barone, V., Accurate excitation energies from timedependent density functional theory: assessing the PBE0 model. J. Chem. Phys. 1999, 111, 28892899.

25. Barone, V.; Cossi, M., Quantum calculation of molecular energies and energy gradients in solution by a conductor solvent model. J. Phys. Chem. A 1998, 102, (11), 1995-2001.

26. Becke, A. D., A new mixing of hartree-fock and local density-functional theories. $J$. Chem. Phys. 1993, 98, 1372-1377.

27. Becke, A. D., Density-functional thermochemistry. III. The role of exact exchange. $J$. Chem. Phys. 1993, 98, 5648-5652.

28. Yanai, T.; Tew, D. P.; Handy, N. C., A new hybrid exchange-correlation functional using the Coulomb-attenuating method (CAM-B3LYP). Chem. Phys. Lett. 2004, 393, (1-3), 51-57.

29. Chai, J. D.; Head-Gordon, M., Long-range corrected hybrid density functionals with damped atom-atom dispersion corrections. Phys. Chem. Chem. Phys. 2008, 10, (44), 6615-6620.

30. Crespo-Hernández, C. E.; Close, D. M.; Gorb, L.; Leszczynski, J., Determination of redox potentials for the Watson-Crick base pairs, DNA nucleosides, and relevant nucleoside analogues. J. Phys. Chem. B 2007, 111, 5386-5395.

31. Ashwood, A.; Steffen, J.; Crespo-Hernández, C. E., Photochemical reactivity of dTPT3: A crucial nucleobase derivative in the development of semisynthetic organisms. J. Phys. Chem. Lett. 2017, 8, 2387-2392.

32. Pollum, M.; Brennan, A.; Steffen, J.; Lam, M.; Crespo-Hernández, C. E., Unintended consequences of expanding the genetic alphabet. J. Am. Chem. Soc. 2016, 138, 11457-11460.

33. Rehm, D.; Weller, A., Kinetics of fluorescence quenching by electron and $\mathrm{H}$-atom transfer. Israel J. Chem. 1970, 8, 259-271.

34. Lakowicz, J. R., Prinicples of Fluorescence Spectroscopy. 3rd ed.; Springer: 2006. 
35. Turro, N. J.; Ramamurthy, V.; Scaiano, J. C., Principles of Molecular Photochemistry: An Introduction. University Science Books: Sausalito, CA, 2009.

36. Schweitzer, C.; Schmidt, R., Physical mechanisms of generation and deactivation of singlet oxygen. Chem. Rev. 2003, 103, 1685-1758.

37. Reichardt, C.; Vogt, R. A.; Crespo-Hernández, C. E., On the origin of ultrafast nonradiative transitions in nitro-polycyclic aromatic hydrocarbons: excited-state dynamics in 1nitronaphthalene. J. Chem. Phys. 2009, 131, 224518.

38. Reichardt, C.; Wen, C.; Vogt, G.; Crespo-Hernández, C. E., Role of intersystem crossing in the fluorescence quenching of 2-aminopurine 2'-deoxyriboside in solution. Photochem. Photobiol. Sci. 2013, 12, 2203.

39. Pollum, M.; Jockusch, S.; Crespo-Hernández, C. E., 2,4-Dithiothymine as a potent UVA chemotherapeutic agent. J. Am. Chem. Soc. 2014, 136, 17930-17933.

40. Carmichael, I.; Hug, G. L., Triplet-triplet absorption spectra of organic molecules in condensed phases. J. Phys. Chem. Ref. Data 1986, 15, 1-32.

41. Reichardt, C.; Crespo-Hernández, C. E., Room-temperature phosphorescence of the DNA monomer analogue 4-thiothymidine in aqueous solution after UVA excitation. J. Phys. Chem.

Lett. 2010, 1, 2239-2243.

42. Pollum, M.; Jockusch, S.; Crespo-Hernández, C. E., Increase in the photoreactivity of uracil derivatives by doubling thionation. Phys. Chem. Chem. Phys. 2015, 17, (41), 2785127861.

43. Schmidt, R.; Tanielian, C.; Dunsbach, R.; Wolff, C., Phenalenone, a universal reference compound for the determination of quantum yields of singlet oxygen $\mathrm{O} 2(1 \Delta \mathrm{g})$ sensitization. $J$. Photochem. Photobiol. A 1994, 79, (1), 11-17.

44. Albert, A.; Brown, B. J., Purine studies. Part I. Stability to acid and alkali. Solubility. Ionization. Comparison with pteridines. J. Chem. Soc. 1954, 2060-2071.

45. Connors, K. A.; Amidon, G. L.; Stella, V. J.; Stella, V. J., Chemical Stability of Pharmaceuticals: A Handbook for Pharmacists. Wiley: 1986.

46. Hemmens, V. J.; Moore, D. E., Photochemical sensitization by azathioprine and its metabolites-I. 6- Mercaptopurine. Photochem. Photobiol. 1986, 43, (3), 247-255.

47. Mitra, A. K.; Narurkar, M. M., Kinetics of azathioprine degradation in aqueous solution. Internat. J. Pharma. 1987, 35, (1-2), 165-171.

48. Pullman, B.; Pullman, A., Electronic aspects of purine tautomerism. Adv. Hetero. Chem. 1971, 13, 77-159.

49. Chenon, M. T.; Pugmire, R. J.; Grant, D. M.; Panzica, R. P.; Townsend, L. B., Carbon-13 magnetic resonace. XXVI. Quantitative determination of the tautomeric populations of certain purines. J. Am. Chem. Soc. 1975, 97, (16), 4636-4642.

50. Broo, A.; Holmén, A., Ab initio MP2 and DFT calculations of geometry and solution tautomerism of purine and some purine derivatives. Chem. Phys. 1996, 211, (1-3), 147-161. 51. Makhyoun, M. A.; Massoud, R. A.; Soliman, S. M., Tautomerism and spectroscopic properties of the immunosuppressant azathioprine. Spectrochimica Acta A 2013, 114, 394-403.

52. Bartl, T.; Zacharová, Z.; Sečkářová, P.; Kolehmainen, E.; Marek, R., NMR quantification of tautomeric populations in biogenic purine bases. Eur. J. Org. Chem. 2009, 1377-1383.

53. Bai, S.; Barbatti, M., On the decay of the triplet state of thionucleobases. Phys. Chem. Chem. Phys. 2017, 19, 12674-12674. 
54. Martínez-Fernández, L.; Corral, I.; Granucci, G.; Persico, M., Competing ultrafast intersystem crossing and internal conversion: a time resolved picture for the deactivation of 6thioguanine. Chem. Sci. 2014, 5, (4), 1336-1347.

55. Sánchez-Rodríguez, J. A.; Mohamadzade, A.; Mai, S.; Ashwood, B.; Pollum, M.; Marquetand, P.; González, L.; Crespo-Hernández, C.; Ullrich, S., 2-Thiouracil intersystem crossing photodynamics studied by wavelength-dependent photoelectron and transient absorption spectroscopies. Phys. Chem. Chem. Phys. 2017, 19, 19756-19766.

56. Koyama, D.; Milner, M. J.; Orr-Ewing, A. J., Evidence for a double well in the first triplet excited state of 2-thiouracil. J. Phys. Chem. B 2017, 121, (39), 9274-9280.

57. Alam, M. M.; Fujitsuka, M.; Watanabe, A.; Ito, O., Photochemical properties of excited triplet state of $6 H$-purine-6-thione investigated by laser flash photolysis. J. Phys. Chem. A 1998, $102,1338-1344$.

58. Borioni, J. L.; Puiatti, M.; Vera, D. M. A.; Pierini, A. B., In search of the best DFT functional for dealing with organic anionic species. Phys. Chem. Chem. Phys. 2017, 19, 91899198.

59. Wang, F.; Hu, Z.; Wang, X.-B.; Sun, Z.; Sun, H., Assessment of DFT methods for the prediction of detachment energies and electronic structures of complex and multiply charged anions. Comp. Theor. Chem. 2021, 1202, 113295.

60. Takaya, T.; Su, C.; De La Harpe, K.; Crespo-Hernández, C. E.; Kohler, B., UV excitation of single DNA and RNA strands produces high yields of exciplex states between two stacked bases. Proc. Natl. Acad. Sci. USA 2008, 105, 10285-10290.

61. Middleton, C. T.; de La Harpe, K.; Su, C.; Law, Y. K.; Crespo-Hernández, C. E.; Kohler, B., DNA excited-state dynamics: from single bases to the double helix. Annu. Rev. Phys. Chem. 2009, 60, 217-239.

62. Al-Mosawi, A. I.; Miller, J. N.; Bridges, J. W., Determination of 6-mercaptopurine and related compounds by phosphorescence spectroscopy. Analyst 1980, 105, 448-454.

63. Ashwood, B.; Ortiz-Rodríguez, L. A.; Crespo-Hernández, C. E., Photochemical relaxation pathways of $\mathrm{S}^{6}$-methylthioinosine and $\mathrm{O}^{6}$-methylguanosine in solution. Faraday Discuss. 2018, 207, 351-374.

64. Zhu, H.; Wang, X.; Ma, R.; Kuang, Z.; Guo, Q.; Xia, A., Intramolecular Charge Transfer and Solvation of Photoactive Molecules with Conjugated Push-Pull Structures. ChemPhysChem 2016, 17, (20), 3245-3251.

65. Zhu, H.; Li, M.; Hu, J.; Wang, X.; Jie, J.; Guo, Q.; Chen, C.; Xia, A., Ultrafast Investigation of Intramolecular Charge Transfer and Solvation Dynamics of Tetrahydro[5]helicene-Based Imide Derivatives. Sci. Rep. 2016, 6, 24313.

66. Mai, S.; Pollum, M.; Martínez-Fernández, L.; Dunn, N.; Marquetand, P.; Corral, I.; Crespo-Hernández, C. E.; González, L., The origin of efficient triplet state population in sulfursubstituted nucleobases. Nature Commun. 2016, 7, 13077.

67. Ortiz-Rodríguez, L. A.; Reichardt, C.; Hoehn, S. J.; Jockusch, S.; Crespo-Hernández, C. E., Detection of the thietane precursor in the UVA formation of the DNA 6-4 photoadduct. Nat. Commun. 2020, 11, 3599. 


\section{Intramolecular Charge Transfer in the Azathioprine Prodrug Quenches Intersystem Crossing to the Reactive Triplet State in 6-Mercaptopurine}

Luis A. Ortiz-Rodríguez, ${ }^{1}$ Glesmarie Ortiz-Zayas, ${ }^{1}$ Marvin Pollum, ${ }^{1}$ Sean J. Hoehn, ${ }^{1}$ Steffen Jockusch, ${ }^{2}$ Carlos E. Crespo-Hernández*,1

${ }^{1}$ Department of Chemistry, Case Western Reserve University, Cleveland, OH 44106

${ }^{2}$ Department of Chemistry, Columbia University, New York, NY 10027

* Corresponding author; E-mail: carlos.crespo@ case.edu; ORCID: 0000-0002-3594-0890

Electronic Supporting Information 


\section{Results}
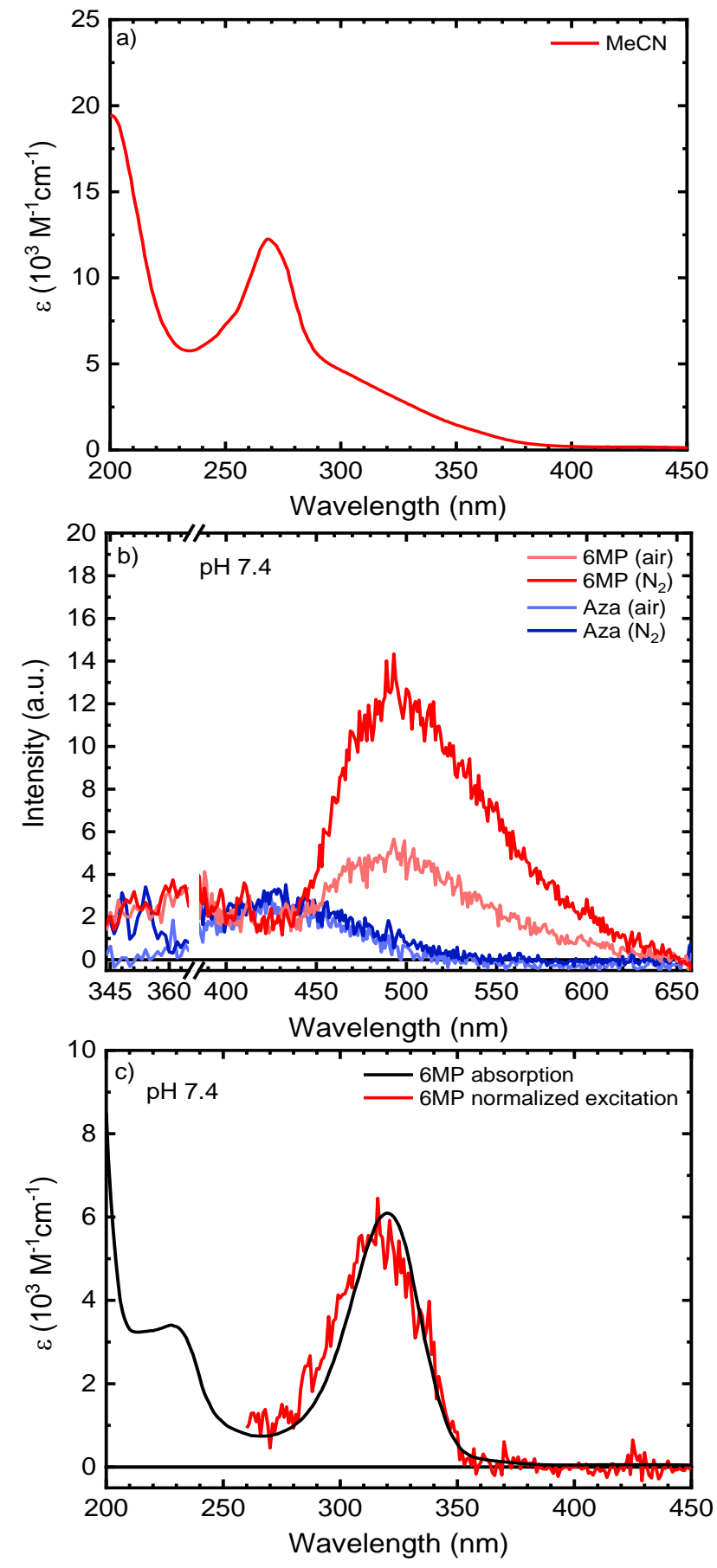

Figure S1. (a) Molar absorption spectrum of Aza in acetonitrile. (b) Emission spectra ( $\lambda_{\mathrm{ex}}=334$ $\mathrm{nm})$ of Aza and 6MP in PBS pH 7.4. (c) Absorption and excitation spectra $\left(\lambda_{\mathrm{em}}=495 \mathrm{~nm}\right)$ of $6 \mathrm{MP}$ in PBS pH 7.4. Excitation spectra $\left(\lambda_{\mathrm{em}}=433 \mathrm{~nm}\right)$ for Aza in PBS pH 7.4 was not reproduced due to extremely low intensity signal. The break in the $\mathrm{x}$-axis of Figure $\mathrm{S} 1 \mathrm{~b}$ conceals the Raman signal. 

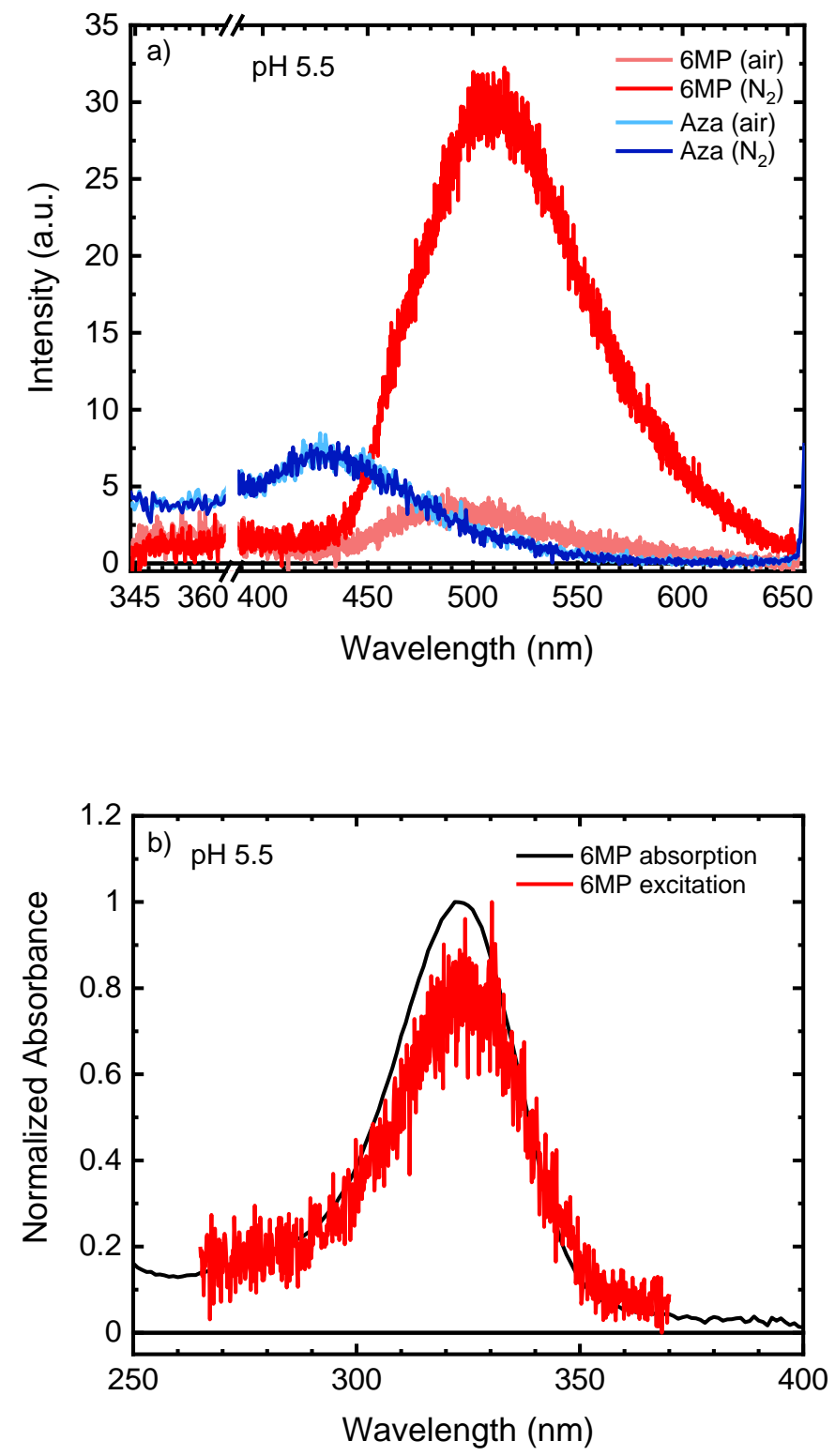

Figure S2. (a) Emission spectra $\left(\lambda_{\mathrm{ex}}=334 \mathrm{~nm}\right)$ of Aza and 6MP in PBS pH 5.5. (b) Absorption and excitation spectra $\left(\lambda_{\mathrm{em}}=495 \mathrm{~nm}\right)$ of $6 \mathrm{MP}$ in PBS pH 5.5. Excitation spectra $\left(\lambda_{\mathrm{em}}=433 \mathrm{~nm}\right)$ for Aza in PBS pH 5.5 was not reproduced due to extremely low intensity signal. The break in the $\mathrm{x}$-axis of Figure S2a conceals the Raman signal. 

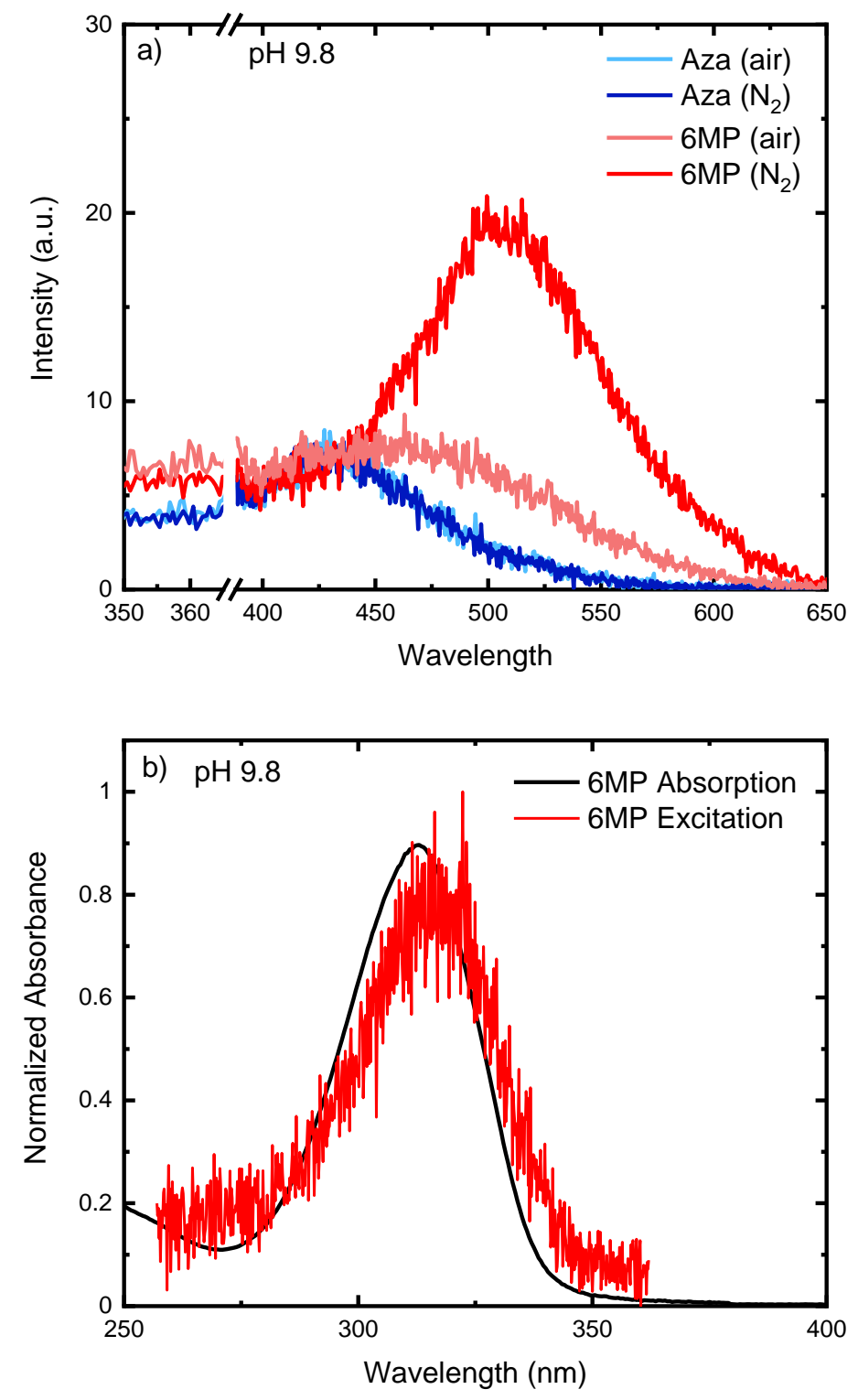

Figure S3. (a) Emission spectra $\left(\lambda_{\mathrm{ex}}=334 \mathrm{~nm}\right)$ of Aza and 6MP in borate buffer $\mathrm{pH}$ 9.8. (b) Absorption and excitation spectra $\left(\lambda_{\mathrm{em}}=510 \mathrm{~nm}\right)$ of $6 \mathrm{MP}$ in borate buffer $\mathrm{pH} 5.5$. Excitation spectra $\left(\lambda_{\mathrm{em}}=425 \mathrm{~nm}\right)$ for Aza in PBS pH 5.5 was not obtained due to extremely low intensity signal. The break in the $x$-axis of Figure S3a conceals the Raman signal. 


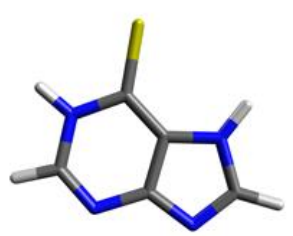

$\mathrm{S}_{1}\left(n \pi^{*}\right)$

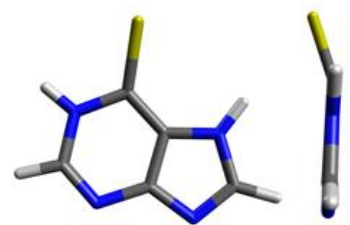

$\mathrm{T}_{2}\left(n \pi^{*}\right)$

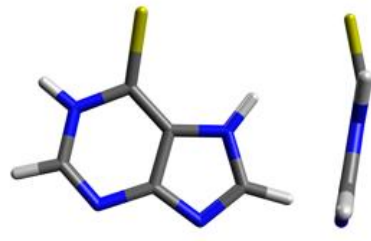

$\mathrm{S}_{2}\left(\pi \pi^{*}\right)$
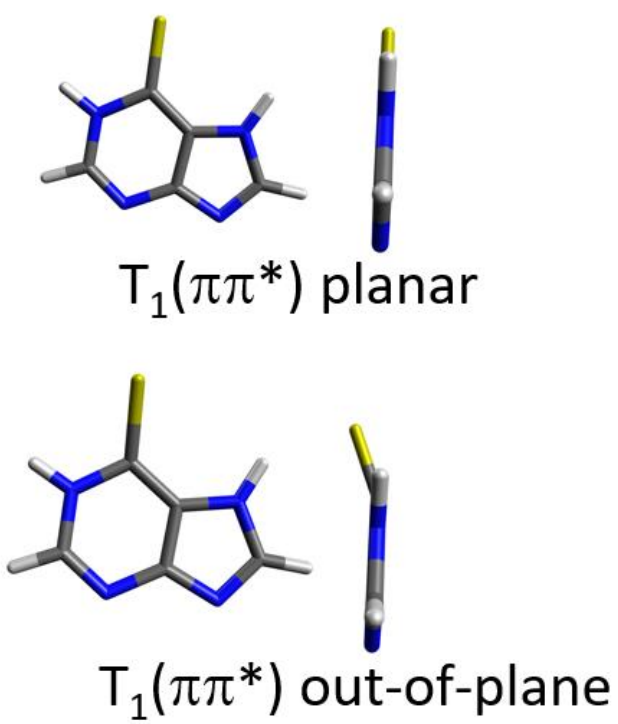

Figure S4. Optimized structures of relevant excited states of neutral 6MP at the TDPBE0/IEFPCM/6-311+G(d,p) in water.

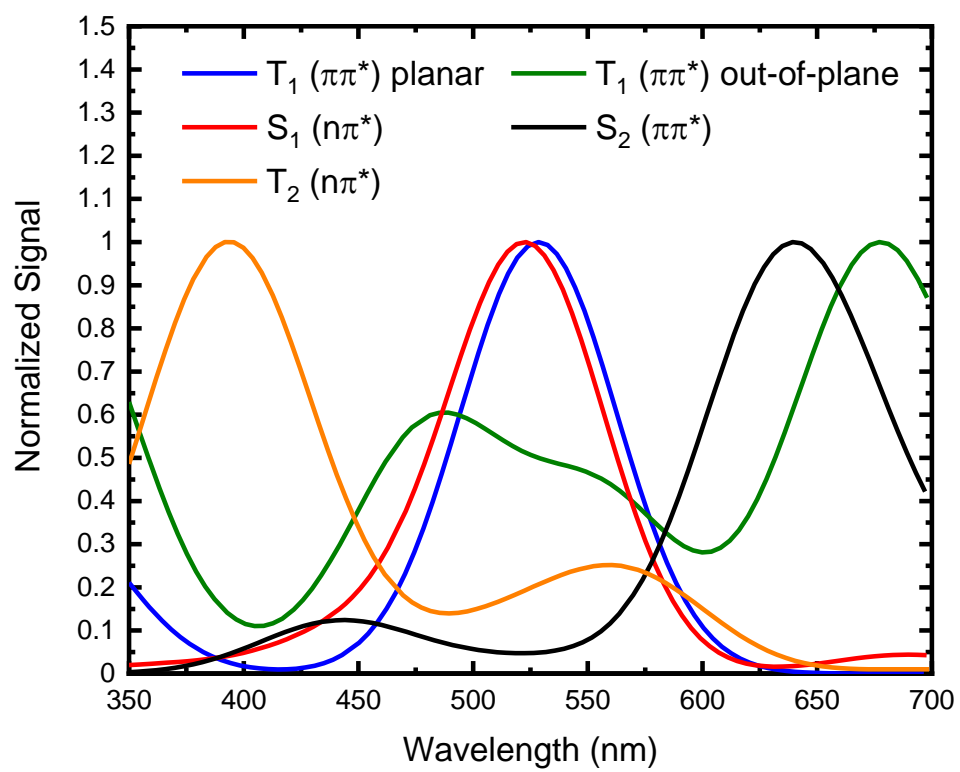

Figure S5. Calculated excited state absorption spectra of relevant excited states of neutral 6MP at the TD-PBE0/CPCM/6-311+G(d,p) in water. The transitions of each excited state absorption spectra were convoluted with a Gaussian function with FWHM $=40 \mathrm{~nm}$. 

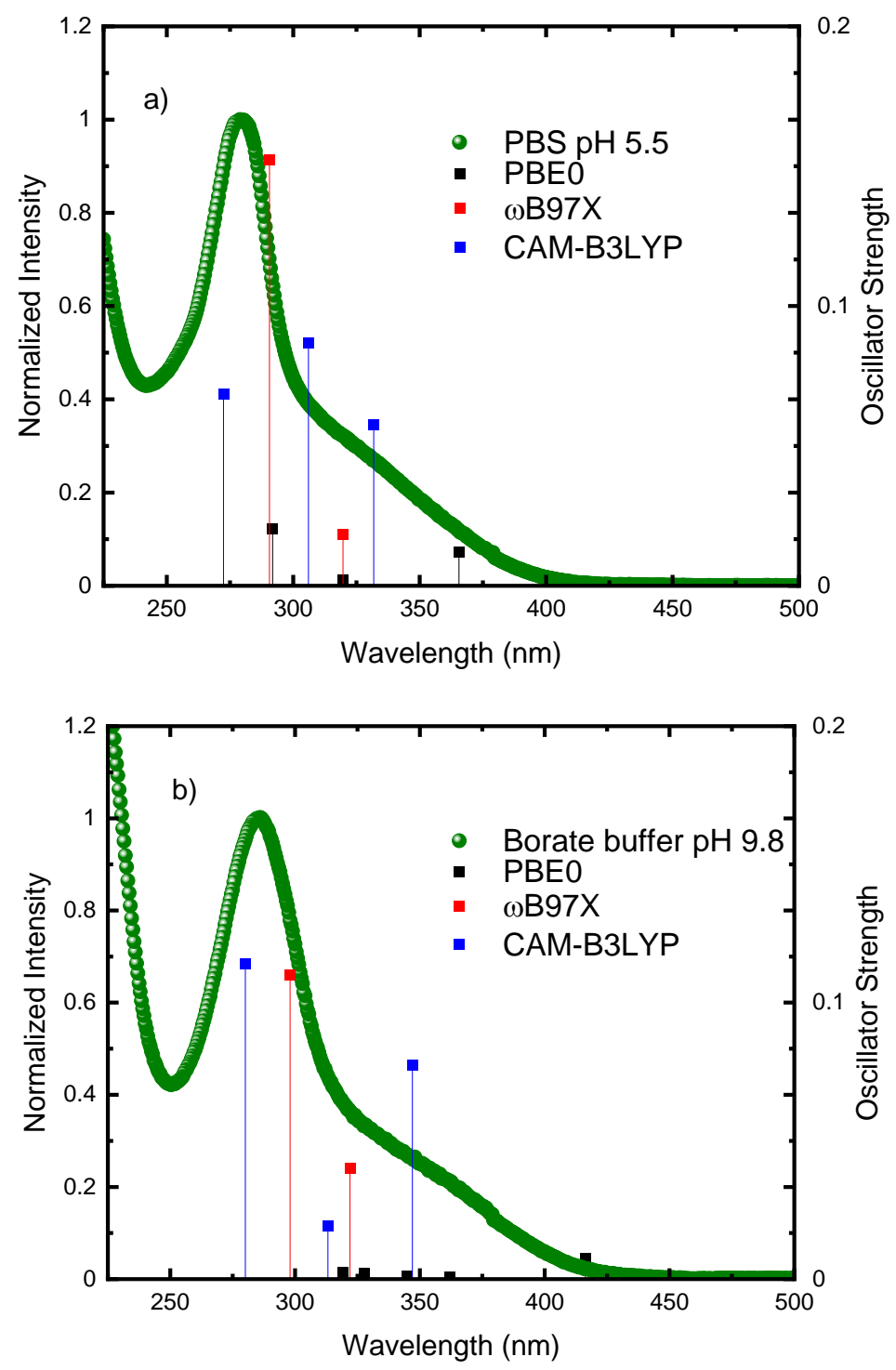

Figure S6. Calculated vertical excitation energies of a) neutral and b) deprotonated Aza overlayed with experimental absorption spectra benchmarking accuracy of the vertical excitation energies. Vertical Excitation energies were calculated at the TD-X/IEFPCM/6-311+G(d,p) level of theory in water, where $\mathrm{X}$ is the functional used. The functionals used were PBE0, CAMB3LYP and $\omega$ B97X. 

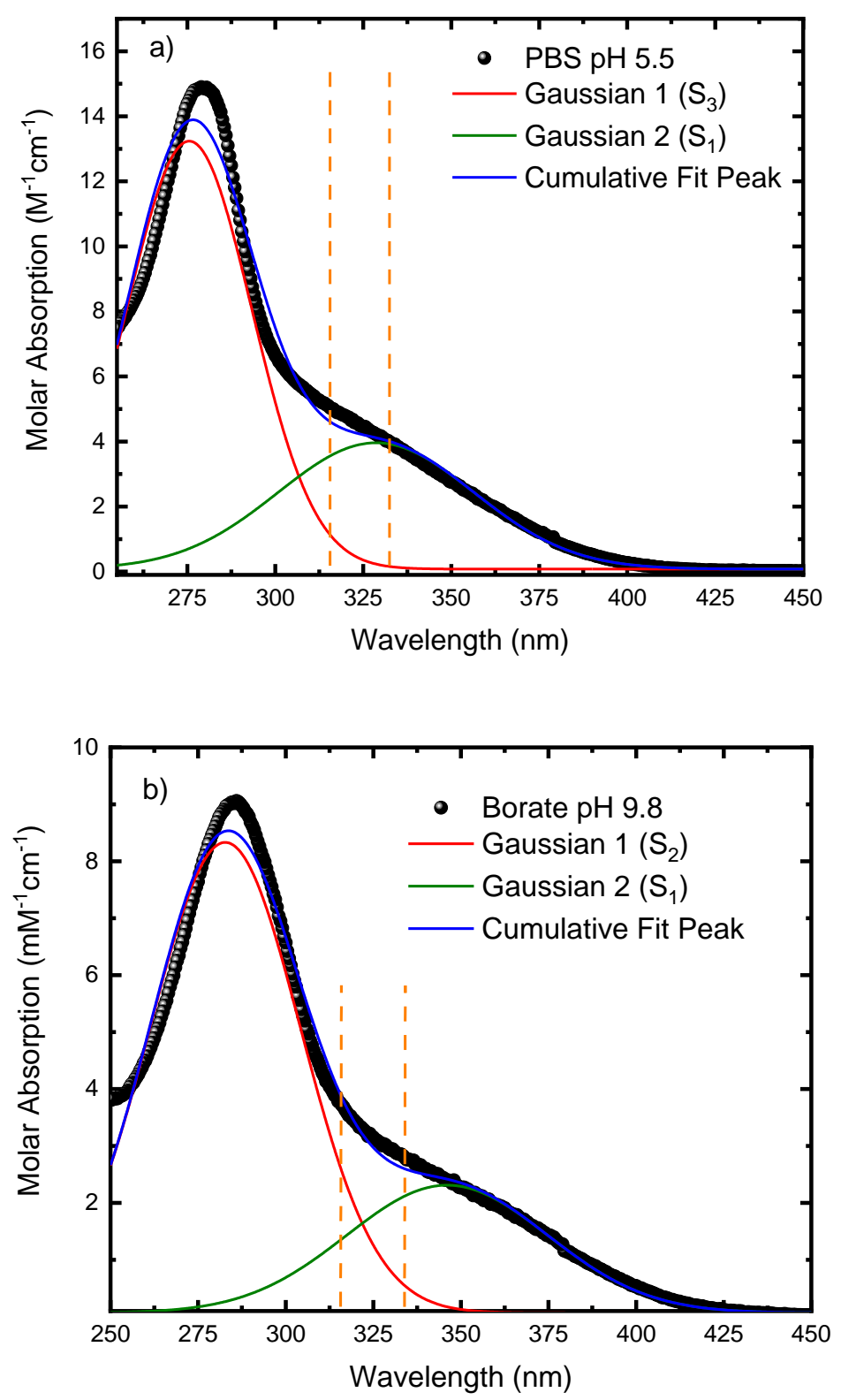

Figure S7. Deconvolution of the two lowest-energy absorption bands in Aza using a linear combination of two Gaussian functions of Aza in a) $\mathrm{pH} 5.5$ and b) $\mathrm{pH}$ 9.8. Excitation wavelengths used in this work are shown as vertical orange dashed lines. For pH 5.5 the two lowest-energy absorption bands were fit with a center wavelength of $276 \mathrm{~nm}$, FWHM of $36 \mathrm{~nm}$ and amplitude of ca. $13.3 \mathrm{mM}^{-1} \mathrm{~cm}^{-1}$, and a center wavelength of $358 \mathrm{~nm}$, FWHM of $56 \mathrm{~nm}$ and amplitude of ca. $4.0 \mathrm{mM}^{-1} \mathrm{~cm}^{-1}$. For $\mathrm{pH} 9.8$ the two lowest-energy absorption bands were fit with a center wavelength of $283 \mathrm{~nm}$, FWHM of $43 \mathrm{~nm}$ and amplitude of ca. $8.3 \mathrm{mM}^{-1} \mathrm{~cm}^{-1}$, and a center wavelength of $346 \mathrm{~nm}$, FWHM of $57 \mathrm{~nm}$ and amplitude of ca. $2.3 \mathrm{mM}^{-1} \mathrm{~cm}^{-1}$. The percent fraction of the population that reach each respective excited singlet state upon the different excitation wavelengths can be determined using equation S1, where $\mathrm{X}$ and $\mathrm{Y}$ represent the respective singlet states: 


$$
\% \text { fraction of population for state } X=\frac{\varepsilon_{X}^{\lambda_{\text {exc }}}}{\varepsilon_{X}^{\lambda_{\text {exc }}}+\varepsilon_{Y}^{\lambda_{\text {exc }}}} \times 100
$$

Based on these calculations, at pH 5.5 (>99\% neutral Aza), when exciting at $334 \mathrm{~nm}, \sim 95 \%$ of the population reaches the $S_{1}$ state and $\sim 5 \%$ the $S_{2}$. At the same $\mathrm{pH}$ but exciting with $316 \mathrm{~nm}$, $\sim 77 \%$ of the population reaches the $\mathrm{S}_{1}$ state and $\sim 23 \%$ the $\mathrm{S}_{2}$. At $\mathrm{pH} 9.8$ (>99\% anionic), when exciting at $334 \mathrm{~nm}, \sim 79 \%$ of the population reaches the $\mathrm{S}_{1}$ state and $\sim 21 \%$ the $\mathrm{S}_{2}$. At the same $\mathrm{pH}$ but exciting with $316 \mathrm{~nm}, \sim 34 \%$ of the population reaches the $S_{1}$ state and $\sim 66 \%$ the $S_{2}$.

Table S1. Vertical ionization energies of anionic 6MP at with three different functionals and MP2.

\begin{tabular}{ll}
\hline Functional & VIE $(\mathrm{kJ} / \mathrm{mol})$ \\
\hline M06 & 341.6 \\
B3PW91 & 341.6 \\
PBE0 & 341.0 \\
MP2 & 420.0 \\
\hline
\end{tabular}



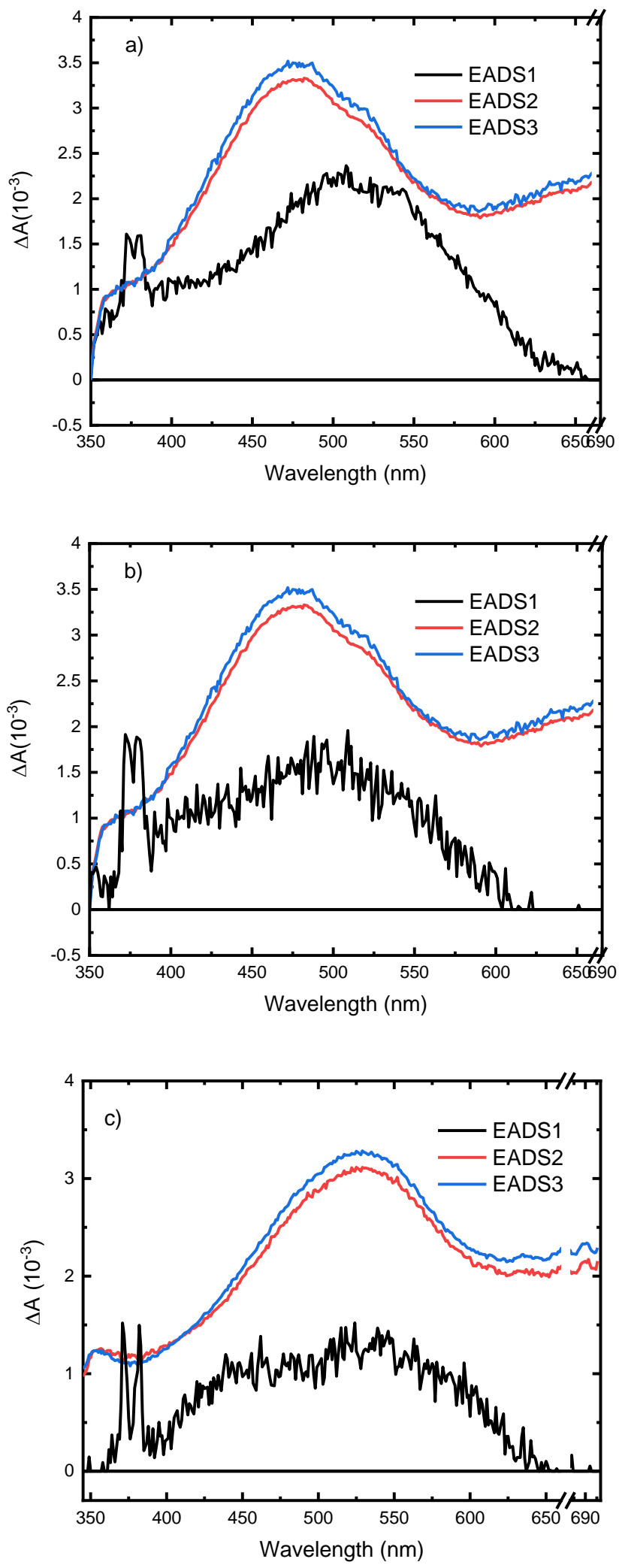

Figure S8. Evolution Associate Difference Spectra (EADS) of 6MP in a) $\mathrm{pH} 5.5, \mathrm{~b}$ ) $\mathrm{pH} 7.4$ and c) $\mathrm{pH} 9.8$. 

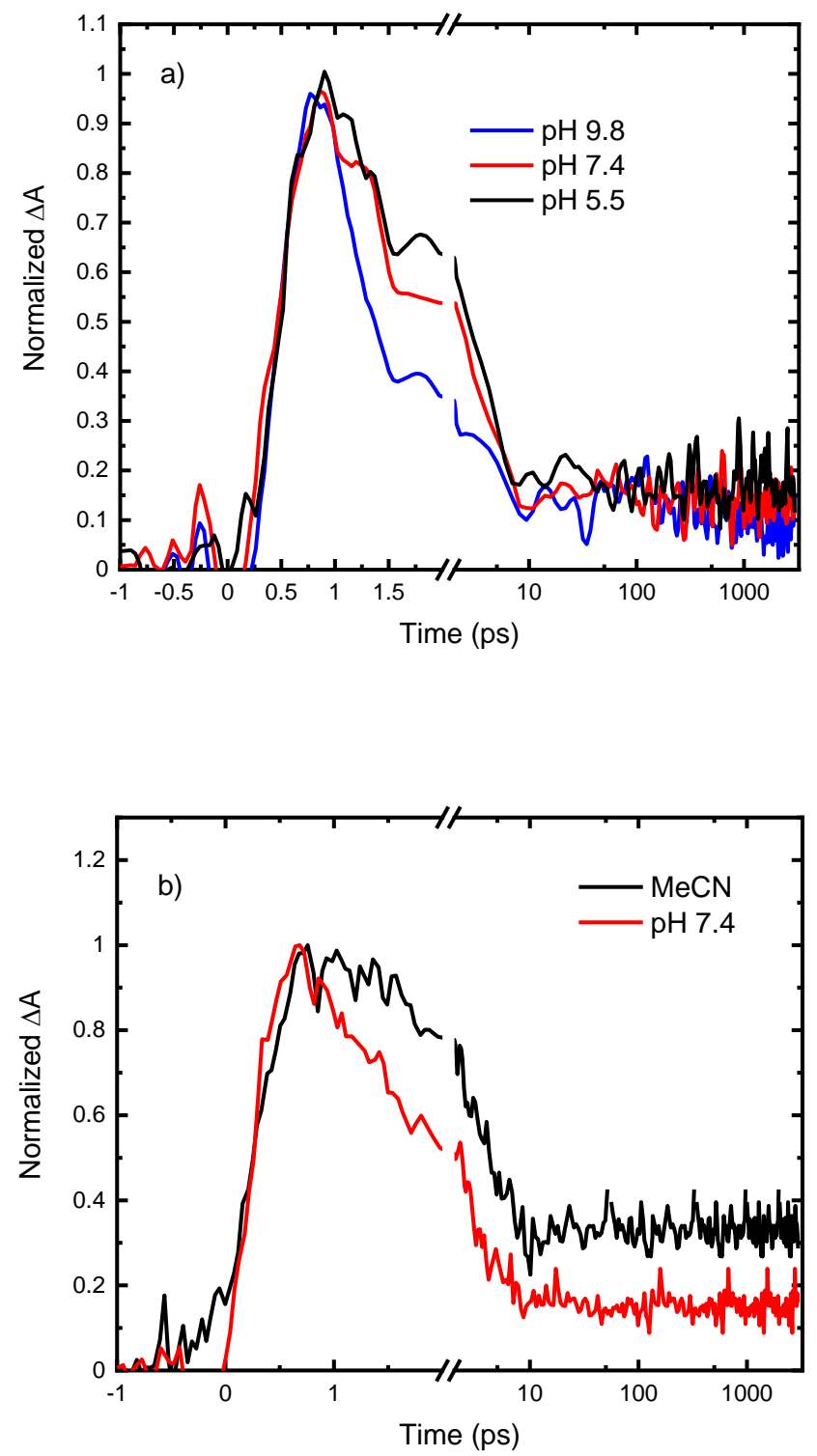

Figure S9. Normalized representative decay traces at $660 \mathrm{~nm}$ of Aza upon excitation at a) $334 \mathrm{~nm}$ and b) $316 \mathrm{~nm}$ 

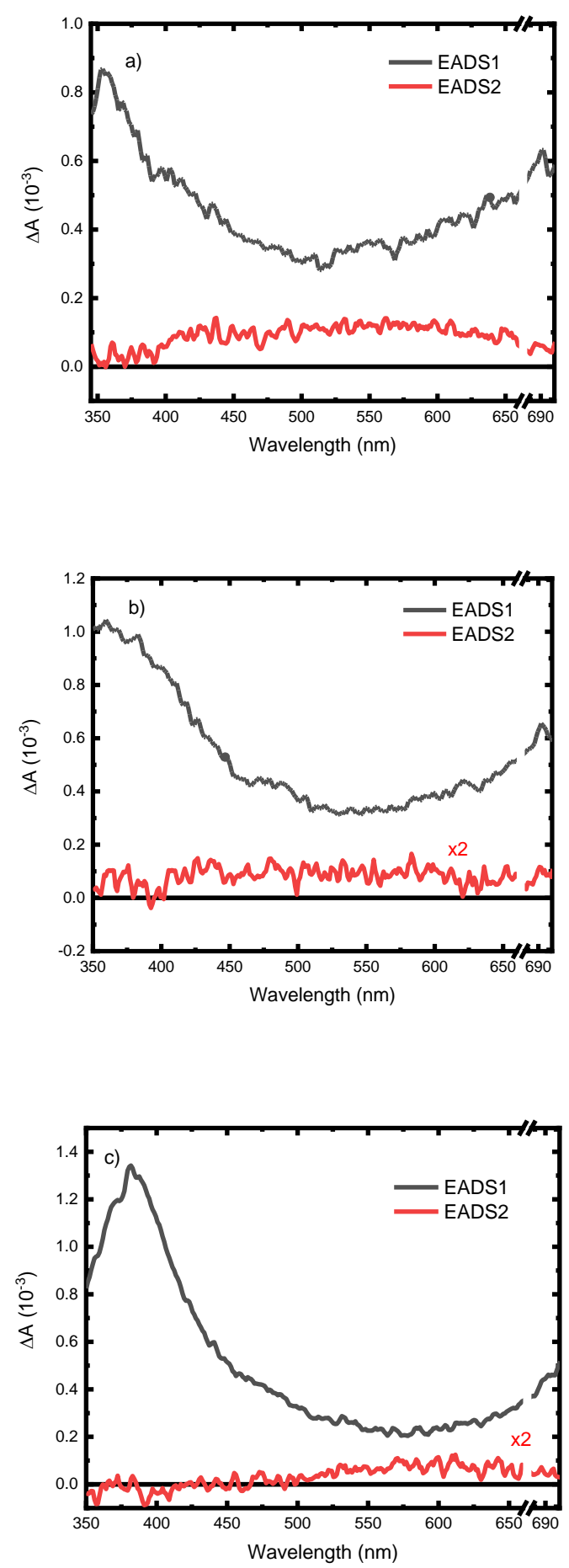

Figure S10. Evolution Associate Difference Spectra (EADS) of Aza in a) $\mathrm{pH} 5.5, \mathrm{~b}$ ) $\mathrm{pH} 7.4$ and c) $\mathrm{pH} 9.8$ 

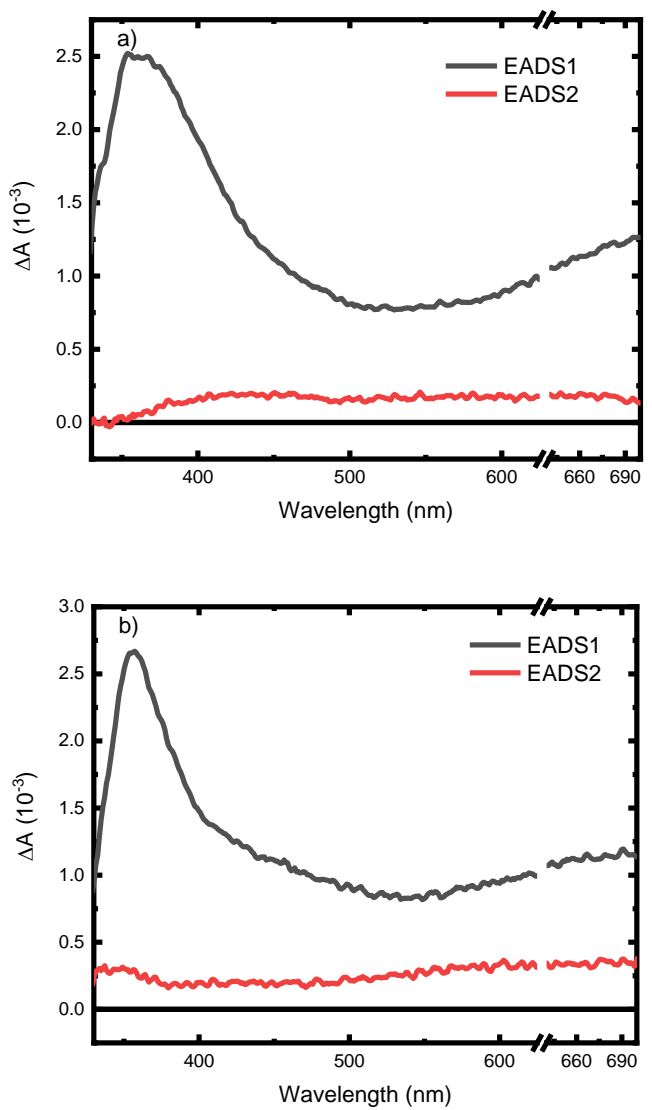

Figure S11. Evolution Associate Difference Spectra (EADS) of Aza upon excitation with 316 $\mathrm{nm}$ in a) $\mathrm{pH} 7.4$ b) MeCN. 


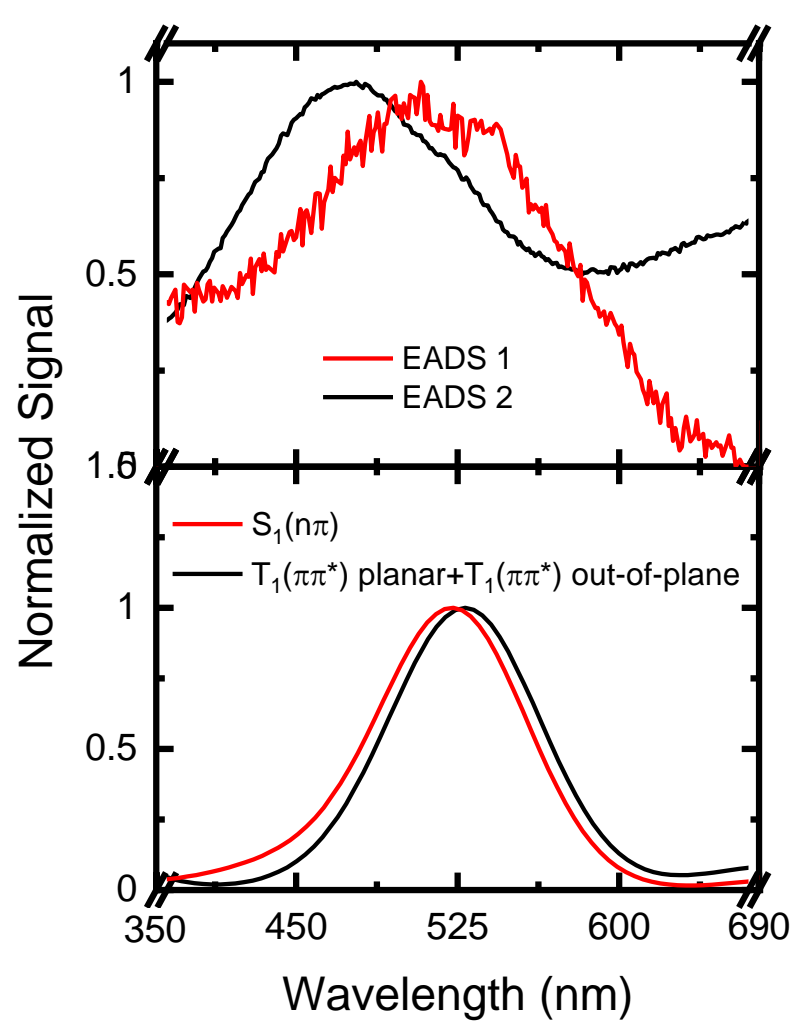

Figure S12. First two evolution-associated difference spectra extracted from the global and target analysis of the transient data of 6MP at pH 5.5 with the excited-state absorption spectra of the $S_{1}\left(n \pi^{*}\right)$ and a linear combination of the excited state absorption spectra of both minima of the $\mathrm{T}_{1}\left(\pi \pi^{*}\right)$. The sum of both spectra was done after normalizing both spectra to the lowest-energy band. 